\title{
On Products of Random Matrices
}

\author{
Natalia Amburg 1,2,3 (D), Aleksander Orlov ${ }^{4, *}$ and Dmitry Vasiliev 1,2,5 (D) \\ 1 A.I. Alikhanov Institute for Theoretical and Experimental Physics of NRC Kurchatov Institute, \\ B. Cheremushkinskaya, 25, 117259 Moscow, Russia; amburg@itep.ru (N.A.); vasiliev@itep.ru (D.V.) \\ 2 Institute for Information Transmission Problems of RAS (Kharkevich Institute), Bolshoy Karetny per. 19, \\ build.1, 127051 Moscow, Russia \\ 3 Moscow Center for Fundamental and Applied Mathematics, 119991 Moscow, Russia \\ 4 Institute of Oceanology, Nahimovskii Prospekt 36, 117997 Moscow, Russia \\ 5 Moscow Institute of Physics and Technology, 141701 Dolgoprudny, Russia \\ * Correspondence: orlovs@ocean.ru
}

Received: 6 July 2020; Accepted: 17 August 2020; Published: 31 August 2020

check for updates

\begin{abstract}
We introduce a family of models, which we name matrix models associated with children's drawings - the so-called dessin d'enfant. Dessins d'enfant are graphs of a special kind drawn on a closed connected orientable surface (in the sky). The vertices of such a graph are small disks that we call stars. We attach random matrices to the edges of the graph and get multimatrix models. Additionally, to the stars we attach source matrices. They play the role of free parameters or model coupling constants. The answers for our integrals are expressed through quantities that we call the "spectrum of stars". The answers may also include some combinatorial numbers, such as Hurwitz numbers or characters from group representation theory.
\end{abstract}

Keywords: random complex and random unitary matrices; matrix models; products of random matrices; Schur polynomial; Hurwitz number; generalized hypergeometric functions; integrable systems

\section{Introduction}

Interest in matrix integrals arose in different contexts and at different times. These are problems of statistics in biology (Wishart), and problems of quantum chaos (Wigner, Dyson, Gorkov, Eliashberg, Efetov), and purely mathematical problems of representation theory (see the textbook [1]). At the end of the previous century, applications were added in the theory of elementary particles ( $t^{\prime}$ Hooft [2]), statistical physics (Migdal, Itsikson and Zuber [3], and Kazakov and Kostov [4]) and string theory (Kazakov and Brezin [5], and Migdal and Gross [6]). Now new applications have been added: in the theory of information transfer and theory of quantum information ([7] or lectures [8] (Ch. 10 on quantum Shannon theory)). We allow ourselves not to provide an ocean of links for each of these areas.

Moving closer to the point, we only refer to works on the use of random matrices in [9], works about products of complex random matrices [10-12], a review [13-16] and works close to ours from a mathematical point of view [17-22].

In the theory of information, the product of the matrices describes the cascade transformation of signals, and averaging over the matrices means introducing interference and noise. The task is to calculate various correlation functions in such models.

We offer a family of models that in a sense can be called exactly solvable (cf. [20]). They are built according to the so-called children's drawings, more precisely, clean children's drawings, which in combinatorics are also called maps. This is a graph drawn on a closed orientable surface, which has the following property: if we cut it along the edges, the surface decomposes into regions homeomorphic to disks (that is, they can be turned into disks by continuous transformation). In addition to this picture, we turn the vertices of the graph into small disks; we call these disks stars. 
The edges of the graph are assigned matrices over which the integration is performed. Depending on the graph we draw, we will get one model or another.

Surprisingly, it turns out that studying the products of random matrices with sources is an easier and more natural task. Writing answers for such integrals turns out to be a faster task if we have these matrices. The absence of these matrices is equivalent to some additional averaging that needs to be specifically monitored.

Writing out some answers requires knowledge of certain combinatorial numbers for which tables exist. In one case, these are the so-called Hurwitz numbers; in the other, these are the characters of representations of a symmetric group. However, sometimes the answers are simplified and written in a fairly simple form-in the form of determinants or in the form of products.

Adding source matrices leads to a variety of interesting relationships with differential operators. We briefly mention this in the Appendix E.

\section{Technical Tools}

\subsection{Partitions; Power Sums and Schur Functions; Hurwitz Numbers}

Here we follow [23]. Further technical details are in Appendices A and B

Partitions. The partition $\lambda=\left(\lambda_{1}, \ldots, \lambda_{k}\right)$ is a set of nonnegative integers $\lambda_{i}$ which are called parts of $\lambda$ and which are ordered as $\lambda_{i} \geq \lambda_{i+1}$. The number of non-vanishing parts of $\lambda$ is called the length of the partition $\lambda$, and will be denoted by $\ell(\lambda)$. The number $|\lambda|=\sum_{i} \lambda_{i}$ is called the weight of $\lambda$. The set of all partitions will be denoted by $\mathrm{Y}$ and the set of partitions of weight $d$ by $\mathrm{Y}_{d}$. Example: the partition $(4,4,1)$ belongs to $Y_{9}$. The length of $(4,4,1)$ is 3 .

We shall use Greek letters for partitions.

Sometimes, it is convenient to use a different notation

$$
\lambda=\left(1^{m_{1}} 2^{m_{2}} 3^{m_{3}} \cdots\right),
$$

where $m_{k}$ is the number of times an integer $k$ occurs as a part of $\lambda$. For instance, $(4,4,1)$ may be written as $\left(1^{1} 4^{2}\right)$. The number

$$
z_{\lambda}=\prod_{k>0} k^{m_{k}} m_{k} !
$$

plays an important role hereinafter.

Example: $z_{(4,4,1)}=z_{\left(1^{1} 4^{2}\right)}=1^{1} 1 ! 4^{2} 2 !=32$.

Partitions can be perceived visually using Young diagrams (YDs): a partition $\lambda$ with parts $\lambda_{1}, \ldots, \lambda_{\ell}$ matches the rows of the corresponding YD of the length $\lambda_{1}, \ldots, \lambda_{\ell}$, respectively; see [23] for details. The weight of $\lambda$ is the area of the YD of $\lambda$.

Power sums. For a matrix $X \in G L_{N}(\mathbb{C})$, we put

$$
p_{k}(X):=\operatorname{tr} X^{k}=x_{1}^{k}+\cdots+x_{N^{\prime}}^{k} \quad k=1,2, \ldots,
$$

which are the Newton sums of its eigenvalues.

Then, for a partition $\Delta=\left(\Delta_{1}, \ldots, \Delta_{k}\right)$, we introduce

$$
\mathbf{p}_{\Delta}(X)=\operatorname{tr}\left(X^{\Delta_{1}}\right) \ldots \operatorname{tr}\left(X^{\Delta_{\ell}}\right) .
$$

We put $\mathbf{p}_{(0)}(X)=1$.

Remark 1. Let us note that $\mathbf{p}_{\Delta}(X)$ is a polynomial in the eigenvalues of $X$ and also a polynomial in entries of $X$. We consider $\mathbf{p}_{\Delta}(X)$ to be a map $G L_{N}(\mathbb{C}) \rightarrow \mathbb{C}$.

The polynomial $\mathbf{p}_{\Delta}$ is a symmetric function of the eigenvalues $x_{1}, \ldots, x_{N}$ and called the power sum labeled with a multi-index $\Delta$. 
If one assigns a degree 1 to each $x_{i}$, then the degree of $\mathbf{p}_{\Delta}(X)$ is $|\Delta|$.

In many problems, the set $p_{1}, p_{2}, \ldots$ is considered as an independent set of complex parameters, which are in no way associated with any matrix. In this case, instead of $\mathbf{p}(X)$ we write simply $\mathbf{p}=\left(p_{1}, p_{2}, \ldots\right)$. The degree of $p_{m}$ is equal to $m$.

Schur functions. Next, let us recall the definition of the Schur polynomial, parametrised by a partition. Consider the equality

$$
e^{\sum_{m>0} \frac{1}{m} z^{m} p_{m}}=1+z p_{1}+z^{2} \frac{p_{1}^{2}+p_{2}}{2}+\cdots=\sum_{m \geq 0} z^{m} s_{(m)}(\mathbf{p})
$$

The polynomials $s_{(m)}$ are called elementary Schur functions. As we can see, $s_{(0)}(\mathbf{p})=1$. Let $\lambda=\left[\lambda_{1}, \lambda_{2}, \ldots\right]$ be a partition, and define the polynomial $s_{\lambda}$ by

$$
s_{\lambda}(\mathbf{p})=\operatorname{det}\left(s_{\left(\lambda_{i}-i+j\right)}(\mathbf{p})\right)_{i, j \geq 1} .
$$

(On the right-hand side, it is assumed that $s_{(m)}=0$ for negative $m$.) Here we define the Schur function as a function of the set of free parameters $\mathbf{p}=\left(p_{1}, p_{2}, \ldots\right)$.

Let us remember that we chose the variables $\mathbf{p}=\left(p_{1}, p_{2}, \ldots\right)$ to be related to a matrix $X \in \mathbb{G L}_{N}(\mathbb{C})$. Then $s_{\lambda}$ is a map $\mathbb{G L}_{N}(\mathbb{C}) \rightarrow \mathbb{C}$; we will write

$$
s_{\lambda}(X):=s_{\lambda}(\mathbf{p}(X)) .
$$

Remark 2. Let us note that $s_{\lambda}(X)$ is a polynomial in entries of $X$ and a symmetric polynomial in the eigenvalues $x_{1}, \ldots, x_{N}$ of degree $d=|\lambda|$.

Remark 3 ([23]). The Schur functions $s_{\lambda}\left(x_{1}, \ldots, x_{N}\right)$, where $\ell(\lambda) \geq N$, form a $\mathbb{Z}$-basis of the space of symmetric polynomials in $x_{1}, \ldots, x_{N}$ of degree $d$.

In terms of the eigenvalues $x_{1}, \ldots, x_{N}$, the Schur function reads as

$$
s_{\lambda}(X)=\frac{\operatorname{det}\left[x_{j}^{\lambda_{i}-i+N}\right]_{i, j \leq N}}{\operatorname{det}\left[x_{j}^{-i+N}\right]_{i, j \leq N}}
$$

for $\ell(\lambda) \leq N$, and vanishes for $\ell(\lambda)>N$. One can see that $s_{\lambda}(x)$ is a symmetric homogeneous polynomial of degree $|\lambda|$ in the variables $x_{1}, \ldots, x_{N}$.

Remark 4. The polynomial $s_{\lambda}$ is the character of the irreducible representation of the $\mathbb{G L}_{N}(\mathbb{C})$ group labeled by $\lambda$.

Character map relation. Relation (4) relates polynomials $s_{\lambda}$ and $\mathbf{p}_{\Delta}$ of the same degree $d=|\lambda|=|\Delta|$. Explicitly, one can write

$$
\mathbf{p}_{\Delta}=\sum_{\lambda \in Y_{d}} \frac{\operatorname{dim} \lambda}{d !} z_{\Delta} \varphi_{\lambda}(\Delta) s_{\lambda}(\mathbf{p})
$$

and

$$
s_{\lambda}(\mathbf{p})=\frac{\operatorname{dim} \lambda}{d !} \sum_{\Delta \in Y_{d}} \varphi_{\lambda}(\Delta) \mathbf{p}_{\Delta} .
$$


The last relation is called the character map relation. Here

$$
\frac{\operatorname{dim} \lambda}{d !}:=\frac{\prod_{i<j \leq N}\left(\lambda_{i}-\lambda_{j}-i+j\right)}{\prod_{i=1}^{N}\left(\lambda_{i}-i+N\right) !}=s_{\lambda}\left(\mathbf{p}_{\infty}\right)
$$

(see Example 1 in Section 1 and Example 5 in Section 3 of Chapter I in [23]), where

$$
\mathbf{p}_{\infty}=(1,0,0, \ldots) .
$$

and where $N \geq \ell(\lambda)$. As one can check, the right-hand side does not depend on $N$. (We recall that $\lambda_{i}=0$ in case $i>\ell(\lambda)$ ). The number $\operatorname{dim} \lambda$ is an integer.

The factors $\varphi_{\lambda}(\Delta)$ satisfy the following orthogonality relations.

$$
\sum_{\lambda \in Y_{d}}\left(\frac{\operatorname{dim} \lambda}{d !}\right)^{2} \varphi_{\lambda}(\mu) \varphi_{\lambda}(\Delta)=\frac{\delta_{\Delta, \mu}}{z_{\Delta}}
$$

and

$$
\left(\frac{\operatorname{dim} \lambda}{d !}\right)^{2} \sum_{\Delta \in Y_{d}} z_{\Delta} \varphi_{\lambda}(\Delta) \varphi_{\mu}(\Delta)=\delta_{\lambda, \mu}
$$

Remark 5. Equality (7) expresses the $\mathbb{G L}_{N}$ character $s_{\lambda}$ in terms of characters $\chi_{\lambda}$ of the symmetric group $S_{d}$ labeled by the same partition $\lambda$ and evaluated on cycle classes $\Delta \in \mathrm{Y}_{d}$. This explains the name of (7). The numbers $\varphi_{\lambda}(\Delta)$ are called the normalized characters:

$$
\chi_{\lambda}(\Delta)=\frac{\operatorname{dim} \lambda}{d !} z_{\Delta} \varphi_{\lambda}(\Delta)
$$

The integer $\operatorname{dim} \lambda$ is the dimension of the representation $\lambda$; that is, $\operatorname{dim} \lambda=\chi_{\lambda}\left(\left(1^{d}\right)\right)$. Equations (10) and (11) are the orthogonality relation for the characters.

Hypergeometric tau functions and determinantal formulas.

Here we follow $[24,25]$. Let $r$ be a function on the lattice $\mathbb{Z}$. Consider the following series

$$
1+r(n+1) x+r(n+1) r(n+2) x^{2}+r(n+1) r(n+2) r(n+3) x^{3}+\cdots=: \tau_{r}(n, x)
$$

Here $n$ is an arbitrary integer. This is just a Taylor series for a function $\tau_{r}$ with a given $n$ written in a form that is as close as possible to typical hypergeometric series. Here $n$ is just a parameter that will be of use later. If as $r$ we take a rational (or trigonometric) function, we get a generalized (or basic) hypergeometric series. Indeed, take

$$
r(n)=\frac{\prod_{i=1}^{p}\left(a_{i}+n\right)}{n \prod_{i=1}^{q}\left(b_{i}+n\right)}
$$

We obtain

$$
\tau_{r}(n, x)=\sum_{m \geq 0} \frac{\prod_{i=1}^{p}\left(a_{i}+n\right)_{m}}{m ! \prod_{i=1}^{q}\left(b_{i}+n\right)_{m}} x^{m}={ }_{p} F_{q}\left(\begin{array}{c}
a_{1}+n, \ldots, a_{p}+n \\
b_{1}+n, \ldots, b_{q}+n
\end{array} \mid x\right)
$$

where

$$
(a)_{n}=a(a+1) \cdots(a+n-1)=\frac{\Gamma(a+n)}{\Gamma(a)}
$$

is the Pochhammer symbol. 
One can prove the following formula which express a certain series over partitions in terms of (12) (see, for instance, [24]):

$$
\tau_{r}(n, X, Y):=\sum_{\lambda} r_{\lambda}(n) s_{\lambda}(X) s_{\lambda}(Y)=\frac{c_{n}}{c_{n-N}} \frac{\operatorname{det}\left[\tau_{r}\left(n-N+1, x_{i} y_{j}\right)\right]_{i, j \leq N}}{\operatorname{det}\left[x_{i}^{N-k}\right]_{i, k \leq N} \operatorname{det}\left[y_{i}^{N-k}\right]_{i, k \leq N}}
$$

where $c_{k}=\prod_{i=0}^{k-1}(r(i))^{i-k}$ and where

$$
r_{\lambda}(n):=\prod_{(i, j) \in \lambda} r(n+j-i), \quad r_{(0)}(n)=1
$$

where the product ranging over all nodes of the Young diagram $\lambda$ is the so-called content product (which has the meaning of the generalized Pochhammer symbol related to $\lambda$ ). Let us test the formula for the simplest case $r \equiv 1$ :

$$
\operatorname{det}[1-X \otimes Y]=\sum_{\lambda} s_{\lambda}(X) s_{\lambda}(Y)=\frac{\operatorname{det}\left[\left(1-x_{i} y_{j}\right)^{-1}\right]_{i, j \leq N}}{\operatorname{det}\left[x_{i}^{N-k}\right]_{i, k \leq N} \operatorname{det}\left[y_{i}^{N-k}\right]_{i, k \leq N}}
$$

Sometimes we also use infinite sets of power sums $\mathbf{p}^{i}=\left(p_{1}^{(i)}, p_{1}^{(i)}, \ldots\right)$ and instead of matrices $X$ and $Y$ set:

$$
\tau_{r}\left(n, \mathbf{p}^{1}, \mathbf{p}^{2}\right):=\sum_{\lambda} r_{\lambda}(n) s_{\lambda}\left(\mathbf{p}^{1}\right) s_{\lambda}\left(\mathbf{p}^{2}\right)
$$

An example $r \equiv 1$ :

$$
\tau_{1}\left(n, \mathbf{p}^{1}, \mathbf{p}^{2}\right):=e^{\sum_{m>0} \frac{1}{m} p_{m}^{(1)} p_{m}^{(2)}}=\sum_{\lambda} s_{\lambda}\left(\mathbf{p}^{1}\right) s_{\lambda}\left(\mathbf{p}^{2}\right)
$$

In case the function $r$ has zeroes, there exists a determinantal representation. Suppose $r(0)$; then $r_{\lambda}(n)=0$ if $\ell(\lambda)>n$. Then

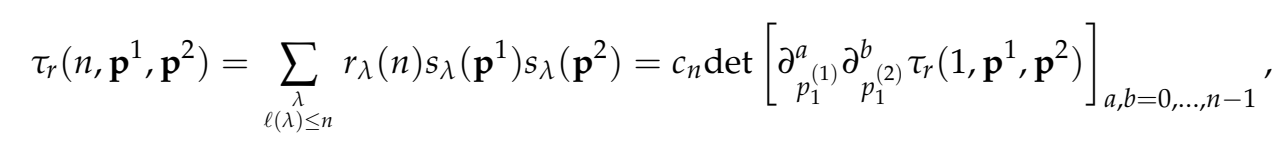

where $c_{k}=\prod_{i=1}^{k-1}(r(i))^{i-k}$, and where

$$
\tau_{r}\left(1, \mathbf{p}^{1}, \mathbf{p}^{2}\right)=1+\sum_{m>0} r(1) \cdots r(m) s_{(m)}\left(\mathbf{p}^{1}\right) s_{(m)}\left(\mathbf{p}^{2}\right) ;
$$

see [26].

In addition, there is the following formula:

$$
\tau_{r}(n, X, \mathbf{p})=\sum_{\lambda} r_{\lambda}(n) s_{\lambda}(X) s_{\lambda}(\mathbf{p})=\frac{\operatorname{det}\left[x_{i}^{N-k} \tau_{r}\left(n-k+1, x_{i}, \mathbf{p}\right)\right]_{i, k \leq N}}{\operatorname{det}\left[x_{i}^{N-k}\right]}
$$

where

$$
\tau_{r}\left(n, x_{i}, \mathbf{p}\right)=1+\sum_{m>0} r(n) \cdots r(n+m) x_{i}^{m} s_{(m)}(\mathbf{p})
$$


Let us test it for $r \equiv 1, \mathbf{p}=\mathbf{p}_{\infty}:=(1,0,0, \ldots)$ :

$$
e^{\operatorname{tr} X}=\sum_{\lambda} s_{\lambda}(X) s_{\lambda}\left(\mathbf{p}_{\infty}\right)=\frac{\operatorname{det}\left[x_{i}^{N-k} e^{x_{i}}\right]_{i, k \leq N}}{\operatorname{det}\left[x_{i}^{N-k}\right]}
$$

There are similar series

$$
\sum_{\lambda} r_{\lambda}(n) s_{\lambda}(X)
$$

which can be written as a Pfaffian [27]; however, we will not use them in the present text.

Some properties of the Schur functions. Let us consider

Lemma 1. For $X \in \mathbb{G L}_{N}$, where $\operatorname{det} X \neq 0$, and for $\lambda=\left(\lambda_{1}, \ldots, \lambda_{N}\right), \ell(\lambda) \leq N$, we have

$$
s_{\lambda}(X) \operatorname{det}\left(X^{\alpha}\right)=s_{\lambda+\alpha}(X)
$$

where $\alpha$ is a nonnegative integer and where $\lambda+\alpha$ denotes the partition with parts $\left(\lambda_{1}+\alpha, \lambda_{2}+\alpha, \ldots, \lambda_{N}+\alpha\right)$, in particular

$$
s_{\lambda}\left(\mathbb{I}_{N}\right)=s_{\lambda+\alpha}\left(\mathbb{I}_{N}\right)
$$

Additionally

$$
\frac{s_{\lambda}\left(\mathbf{p}_{\infty}\right)}{s_{\lambda+\alpha}\left(\mathbf{p}_{\infty}\right)}=\prod_{i=1}^{N} \frac{\Gamma\left(h_{i}+1+\alpha\right)}{\Gamma\left(h_{i}+1\right)}=\frac{(N+\alpha)_{\lambda}}{(N)_{\lambda}}
$$

Hurwitz number. Let $\mathrm{E} \leq 2$ be an integer and $\Delta^{1}=\left(\Delta_{1}^{1}, \Delta_{2}^{1}, \ldots\right), \ldots, \Delta^{k}=\left(\Delta_{1}^{k}, \Delta_{2}^{k}, \ldots\right) \in \mathrm{Y}_{d}$. One can show that

$$
H_{\mathrm{E}}\left(\Delta^{1}, \ldots, \Delta^{k}\right)=\sum_{\lambda \in \mathrm{Y}_{d}}\left(\frac{\operatorname{dim} \lambda}{d !}\right)^{\mathrm{E}} \varphi_{\lambda}\left(\Delta^{1}\right) \cdots \varphi_{\lambda}\left(\Delta^{k}\right)
$$

is a rational number. This number is called the Hurwitz number, which is a popular combinatorial object in many fields of mathematics (see, for instance, [28,29]) and also in physics [30]. The explanations of the geometrical and combinatorial meanings of Hurwitz numbers may be found in Appendices C and D. We also need a weighted Hurwitz number

$$
H_{\mathrm{E}}\left(\Delta^{1}, \ldots, \Delta^{k} \mid m\right)=\sum_{\lambda \in \mathrm{Y}_{d}}\left(\frac{\operatorname{dim} \lambda}{d !}\right)^{\mathrm{E}} \varphi_{\lambda}\left(\Delta^{1}\right) \cdots \varphi_{\lambda}\left(\Delta^{k}\right)\left(\frac{1}{(N)_{\lambda}}\right)^{m}
$$

\subsection{Mixed Ensembles of Random Matrices}

A complex number $\mathbb{E}_{n_{1}, n_{2}}\{f\}$ is the notation of the expectation values of a function $f$ which depends on the entries of matrices $Z_{1}, \ldots, Z_{n_{1}} \in \mathbb{G L}_{N}(\mathbb{C})$ and of matrices $U_{1}, \ldots, U_{n_{2}} \in \mathbb{U}_{N}$ :

$$
\begin{gathered}
\mathbb{E}_{n_{1}, n_{2}}\{f\}=\int f\left(Z_{1}, \ldots, Z_{n_{1}}, U_{1}, \ldots, U_{n_{2}}\right) d \Omega_{n_{1}, n_{2}}, \\
d \Omega_{n_{1}, n_{2}}=\prod_{i=1}^{n_{1}} d \mu\left(Z_{i}\right) \prod_{i=1}^{n_{2}} d_{*} U_{i}
\end{gathered}
$$

where $d_{*} U_{i}\left(i=1, \ldots, n_{2}\right)$ is the Haar measure on $\mathbb{U}_{N}$ and where

$$
d \mu\left(Z_{i}\right)=c \prod_{a, b} e^{-\hbar^{-1}\left|\left(Z_{i}\right)_{a, b}\right|^{2}} d^{2}\left(Z_{i}\right)_{a, b}
$$

is the Gaussian measure. Here $\hbar$ is a parameter; usually it is chosen to be $N^{-1}$. 
Each set of $Z_{i}$ and $d \mu\left(Z_{i}\right)$ is called complex Ginibre ensemble and the whole set $Z_{1}, \ldots, Z_{n_{1}}$ and $d \mu\left(Z_{1}\right), \ldots, d \mu\left(Z_{n_{1}}\right)$ are called $n_{1}$ independent complex Ginibre ensembles. The set $U_{1}, \ldots, U_{n_{2}}$ and the measure $d_{*} U_{1}, \ldots, d_{*} U_{n_{2}}$ are called $n_{2}$ independent circular ensembles. We assume each $\int d_{*} U_{i}=\int d \mu\left(Z_{i}\right)=1$.

The ensemble of the matrices $Z_{1}, \ldots, Z_{n_{1}}, U_{1}, \ldots, U_{n_{2}}$ together with the probability measure $d \Omega_{n_{1}, n_{2}}$ (that is, with expectation values defined by (29)) we call a $\left(n_{1}, n_{2}\right)$ mixed ensemble. We will consider mixed ensembles with $n=n_{1}+n_{2}$ random matrices.

\subsection{Integrals of Schur Functions and Integrals of Power Sums}

In what follows we study expectation values of Schur functions and of power sums. We need four key lemmas.

These lemmas should be known in parts corresponding to Schur functions, but at the moment I have not found all the lemmas along with the proofs, so I will try to fill this gap.

In the Lemmas 2-5 below $A$ and $B$ are $N \times N$ complex matrices; this point is the key.

Everywhere in this section, $d=1, \ldots, N$.

Lemma 2. (I) For any $\lambda \in \mathrm{Y}_{d}$ we have

$$
\mathbb{E}_{1,0}\left\{s_{\lambda}\left(Z A Z^{\dagger} B\right)\right\}=\hbar^{d} \frac{s_{\lambda}(A) s_{\lambda}(B)}{s_{\lambda}\left(\mathbf{p}_{\infty}\right)}
$$

where $s_{\lambda}\left(\mathbf{p}_{\infty}\right)$ is given in (8).

(II) Equivalently, for any $\Delta \in \mathrm{Y}_{d}$ we have

$$
\mathbb{E}_{1,0}\left\{\mathbf{p}_{\Delta}\left(Z A Z^{\dagger} B\right)\right\}=z_{\Delta} \hbar^{d} \sum_{\Delta^{a}, \Delta^{b} \in Y_{d}} H_{2}\left(\Delta, \Delta^{a}, \Delta^{b}\right) \mathbf{p}_{\Delta^{a}}(A) \mathbf{p}_{\Delta^{b}}(B)
$$

where

$$
H_{2}\left(\Delta, \Delta^{a}, \Delta^{b}\right)=\sum_{\lambda \in Y_{d}}\left(\frac{\operatorname{dim} \lambda}{d !}\right)^{2} \varphi_{\lambda}(\Delta) \varphi_{\lambda}\left(\Delta^{a}\right) \varphi_{\lambda}\left(\Delta^{b}\right)
$$

is the three-point Hurwitz number (27).

Note that Hurwitz numbers do not depend on the order of its arguments, in particular, for (33), $H_{2}\left(\Delta, \Delta^{a}, \Delta^{b}\right)=H_{2}\left(\Delta, \Delta^{b}, \Delta^{a}\right)=\cdots=H_{2}\left(\Delta^{b}, \Delta^{a}, \Delta\right)$.

Proof. (i) Relation (31) is known for Hermitian $A, B$; see [23], Chap. VII, Section 5, Example 5. Taking into account Remark 2, we see that both sides of (31) can be analytically continued as functions of matrix entries of $A$ and $B$. Therefore, (31) is correct for $A, B \in \mathbb{G L}_{N}(\mathbb{C})$.

(ii) Relation (32) follows from (31) and vice versa. To see this we use (10). For example, let us get (32) from (31). We replace all Schur functions in (31) with power sums in accordance with (7). Then we multiply the both sides of relation (31) by $\varphi_{\lambda}(\Delta) \operatorname{dim} \lambda$, then we sum over $\lambda$. Then the first orthogonality relation (10) and (27) results in (32).

(iii) The alternative way to prove the Lemma is to start with the left-hand side of (32), where $A, B \in \mathbb{G L}_{N}(\mathbb{C})$. Such integrals were considered in [31] in the context of generating of Hurwitz numbers (see Appendix $C$ ). Using the results of [31] we state that it is equal to the right-hand side of (32), where $H_{2}\left(\Delta, \Delta^{a}, \Delta^{b}\right)$ is the three-point Hurwitz number given by (27), namely, to right-hand side of (33). Then with the help of orthogonality relation (10), we derive (31), where $A, B \in \mathbb{G L}_{N}(\mathbb{C})$.

Remark 6. Regarding the last point (iii): The volume of the article does not allow describing the construction of work [31,32]. In short: The derivation of the formula (33), and the derivation of more general formulas (68) below, are based on the application of Wick's theorem and the realization of the fact that each Wick pairing corresponds to a certain gluing of surfaces from polygons: the surface obtained in the first order of the perturbation theory $(|\Delta|=d=1$ ) is basic. (For instance, the torus can be obtained from a rectangular by gluing the opposite sides; 
see (a) and (b) in Figure 3.) The surfaces obtained in the following orders $(d>1)$ are the surfaces which cover the basic one. In this case, the Hurwitz numbers simply give a weighted number of possible covering surfaces, where the Young diagrams correspond to the so-called branching profiles. The basic surface in case (33) is a sphere, glued from the 2-gon; see Figure $2 b$ below where $X_{1}=Z, X_{-1}=Z^{\dagger}, C_{1}=A, C_{-1}=B$.

Examples of (33). Suppose $\Delta=(2)$ on the left-hand side of (33). It is known that $H_{2}\left((2), \Delta^{a}, \Delta^{b}\right)=1 / 2$ in two cases-where $\Delta^{a}=(2), \Delta^{b}=(1,1)$ and where $\Delta^{a}=(1,1), \Delta^{b}=(2)$ - and it is zero otherwise. Additionally $z_{(2)}=2$, see (1). Thus,

$$
\mathbb{E}_{1,0}\left\{\operatorname{tr}\left(\left(Z A Z^{\dagger} B\right)^{2}\right)\right\}=\hbar^{2} \operatorname{tr}\left(A^{2}\right)(\operatorname{tr} B)^{2}+\hbar^{2}(\operatorname{tr} A)^{2} \operatorname{tr}\left(B^{2}\right) .
$$

Next, suppose $\Delta=(1,1)$. As we know, $H_{2}((1,1),(2),(2))=1 / 2$ and vanishes for different choices of $\Delta^{a}, \Delta^{b}$, and $z_{(1,1)}=2$; see (1); therefore,

$$
\mathbb{E}_{1,0}\left\{\left(\operatorname{tr} Z A Z^{\dagger} B\right)^{2}\right\}=\hbar^{2} \operatorname{tr}\left(A^{2}\right) \operatorname{tr}\left(B^{2}\right) .
$$

Corollary 1. Suppose $\operatorname{det} A \neq 0, \operatorname{det} B \neq 0$, and $\lambda=\left(\lambda_{1}, \ldots, \lambda_{N}\right), \ell(\lambda) \leq N$. We get

$$
\mathbb{E}_{1,0}\left\{s_{\lambda}\left(Z A Z^{\dagger} B\right) \operatorname{det}\left(\left(Z^{\dagger} Z\right)^{p_{0}}\right)\right\}=\hbar^{d+p_{0} N} \frac{s_{\lambda}(A) s_{\lambda}(B)}{s_{\lambda}\left(\mathbf{p}_{\infty}\right)} \frac{\left(N+p_{0}\right)_{\lambda}}{(N)_{\lambda}}
$$

In particular,

$$
\mathbb{E}_{1,0}\left\{s_{\lambda}\left(Z A Z^{\dagger}\right) \operatorname{det}\left(\left(Z^{\dagger} Z\right)^{p_{0}}\right)\right\}=\hbar^{d+p_{0} N} s_{\lambda}(A)\left(N+p_{0}\right)_{\lambda}
$$

Proof. In case $p_{0}$ is a natural number the proof is as follows:

$$
s_{\lambda}\left(Z A Z^{\dagger} B\right) \operatorname{det}\left(\left(Z A Z^{\dagger} B\right)^{p_{0}}\right)=s_{\lambda}\left(Z A Z^{\dagger} B\right) \operatorname{det}\left(\left(Z Z^{\dagger}\right)^{p_{0}}\right) \operatorname{det}\left((A)^{p_{0}}\right) \operatorname{det}\left((B)^{p_{0}}\right)=s_{\lambda+p_{0}}\left(Z A Z^{\dagger} B\right)
$$

and from Lemma 1: where $\lambda+p_{0}=\left(\lambda_{1}+p_{0}, \lambda_{2}+p_{0}, \ldots, \lambda_{N}+p_{0}\right), \ell\left(\lambda^{\prime}\right)=N$. We have

$$
\frac{s_{\lambda}\left(\mathbf{p}_{\infty}\right)}{s_{\lambda^{\prime}}\left(\mathbf{p}_{\infty}\right)}=\prod_{i=1}^{N} \frac{\Gamma\left(h_{i}+1+p_{0}\right)}{\Gamma\left(h_{i}+1\right)}=\frac{\left(N+p_{0}\right)_{\lambda}}{(N)_{\lambda}}
$$

As we see he right-hand side can by analytically continued as the function of $p_{0}$.

Lemma 3. (I) Suppose $\lambda \in \mathrm{Y}_{d}$, and let $v$ be any partition. We have

$$
\mathbb{E}_{1,0}\left\{s_{\lambda}(Z A) s_{v}\left(Z^{\dagger} B\right)\right\}=\hbar^{d} \delta_{\lambda v} \frac{s_{\lambda}(A B)}{s_{\lambda}\left(\mathbf{p}_{\infty}\right)}
$$

where $s_{\lambda}\left(\mathbf{p}_{\infty}\right)$ is given in (8).

(II) Equivalently, let $\Delta^{a} \in \mathrm{Y}_{d}$ and $\Delta^{b} \in \mathrm{Y}_{d^{\prime}}$. Then

$$
\mathbb{E}_{1,0}\left\{\mathbf{p}_{\Delta^{a}}(Z A) \mathbf{p}_{\Delta^{b}}\left(Z^{\dagger} B\right)\right\}=z_{\Delta^{a} z_{\Delta^{b}} \delta_{d, d^{\prime}} \hbar^{d}} \sum_{\Delta \in Y_{d}} H_{2}\left(\Delta^{a}, \Delta^{b}, \Delta\right) \mathbf{p}_{\Delta}(A B),
$$

where $H_{\mathbb{C P}^{1}}\left(\Delta, \Delta^{a}, \Delta^{b}\right)$ is given by (33).

The identity (37) is well-known; for instance, see [23]. Relation (38) was proven independently in [31]. To obtain (37) from (38) we replace power sums under the integral with the left-hand side of (6) and use (27) and the orthogonality relations (10) and (11). 
Denote $N \times N$ identity matrix $\mathbb{I}_{N}$. The following equality is known (see [23]: combine Examples 4 and 5 of Section 3 and Example 1 from Section I of Chapter I):

$$
s_{\lambda}\left(\mathbb{I}_{N}\right)=(N)_{\lambda} s_{\lambda}\left(\mathbf{p}_{\infty}\right) .
$$

Here

$$
(N)_{\lambda}:=(N)_{\lambda_{1}}(N-1)_{\lambda_{2}} \cdots(N-\ell+1)_{\lambda_{\ell^{\prime}}}
$$

where $\lambda=\left(\lambda_{1}, \ldots, \lambda_{\ell}\right), \lambda_{\ell} \neq 0$ and where $(a)_{k}:=a(a+1) \cdots(a+k-1)=\frac{\Gamma(a+k)}{\Gamma(a)}$ is the Pochhammer symbol.

Lemma 4. For any $\lambda \in \mathrm{Y}_{d}$, we have

$$
\mathbb{E}_{0,1}\left\{s_{\lambda}\left(U A U^{-1} B\right)\right\}=\frac{s_{\lambda}(A) s_{\lambda}(B)}{s_{\lambda}\left(\mathbb{I}_{N}\right)} .
$$

The proof is contained in [23] Chap VII, Section 5, Example 3.

Lemma 5. Suppose $\lambda \in \mathrm{Y}_{d}$. For any $\mu$, we get

$$
\mathbb{E}_{0,1}\left\{s_{\mu}(U A) s_{\lambda}\left(U^{-1} B\right)\right\}=\frac{s_{\lambda}(A B)}{s_{\lambda}\left(\mathbb{I}_{N}\right)} \delta_{\mu, \lambda} .
$$

The proof follows from relations (1) and (3) Chap VII, Section 5, Example 1 in [23].

Remark 7. Formula (41) gives the fastest derivation of the famous formula HCIZ:

$$
\int e^{\alpha \operatorname{tr} U A U^{+} B} d_{*} U=c \frac{\operatorname{det}\left[e^{a_{i} b_{j}}\right]_{i, j}}{\prod_{i>j}\left(a_{i}-a_{j}\right)\left(b_{i}-b_{j}\right)}
$$

Indeed, we use (19) where $\mathbf{p}^{1}=(1,0,0, \ldots)=: \mathbf{p}_{\infty}$ and $\mathbf{p}^{2}=\left(p_{1}^{(2)}, p_{2}^{(2)}, \ldots\right)$ with $p_{m}^{(2)}=\operatorname{tr}\left(\left(U A U^{\dagger} B\right)^{m}\right) ;$ thus,

$$
e^{\alpha \operatorname{tr} U A U^{\dagger} B}=\sum_{\lambda} \alpha^{|\lambda|} s_{\lambda}\left(\mathbf{p}_{\infty}\right) s_{\lambda}\left(U A U^{\dagger} B\right)
$$

which gives

$$
\int e^{\alpha \operatorname{tr} U A U^{\dagger} B} d_{*} U=\sum_{\lambda} \alpha^{|\lambda|} \frac{s_{\lambda}\left(\mathbf{p}_{\infty}\right) s_{\lambda}(A) s_{\lambda}(B)}{s_{\lambda}\left(\mathbb{I}_{N}\right)}=\sum_{\lambda} \frac{\alpha^{|\lambda|}}{(N)_{\lambda}} s_{\lambda}(A) s_{\lambda}(B)
$$

which has the form (15). In a similar waym one evaluates $\int\left(1-\alpha U A U^{\dagger} B\right)^{-a} d_{*} U$ and a number of integrals; see, for instance [26].

The expectation values $\mathbb{E}_{0,1}\left\{\mathbf{p}_{\Delta}\left(A U B U^{-1}\right)\right\}$ and $\mathbb{E}_{0,1}\left\{\mathbf{p}_{\Delta^{a}}(A U) \mathbf{p}_{\Delta^{b}}(B U+)\right\}$ can also be expressed in terms of Hurwitz numbers, but this requires a lot of space, and we will not do this; see the last section [31] for details.

\section{Our Models. Products of Random Matrices We Choose}

\subsection{Preliminary. On the Products of Random Matrices}

In a number of articles the spectral correlation functions of certain products of were were studied. These are products

$$
Z_{1} Z_{1}^{+} \cdots Z_{n} Z_{n}^{+}
$$




$$
Z_{1} \cdots Z_{n}\left(Z_{1} \cdots Z_{n}\right)^{\dagger}
$$

or, a pair of products

$$
Z_{1} \cdots Z_{n}, \quad\left(Z_{1} \cdots Z_{n}\right)^{\dagger}
$$

see [10-12].

Similar products in which complex matrices are replaced by unitary and certain generalizations have also been considered [16,21,22,33-35].

Below we suggest a generalization of these models (see Examples 18 and 16 respectively for (43)-(45)). We call it matrix models related to dessin d'enfants, more precisely, to the so-called clean dessin d'enfants (see [36]).

This is a child's drawing of a "constellation", like the Greek constellation, which is painted in the sky with stars, and the sky is a surface with a chosen Euler characteristic.

See below for a more accurate description.

As a part of dessin d'enfants we will need the following modification:

(1) We shall consider mixed ensembles, and in what follows, $X_{ \pm i}$ denotes either $Z_{ \pm i}$, where $Z_{-i}=Z_{i}^{\dagger}$, or $U_{ \pm i}$, where $U_{-i}=U_{i}^{\dagger}$.

(2) We need additional data, which we call source matrices $C_{ \pm 1}, \ldots, C_{ \pm n} \in \mathbb{G L}_{N}(\mathbb{C})$. Each random matrix $X_{i}$ enters only in combination $X_{i} C_{i}$ :

$$
X_{i} \rightarrow X_{i} C_{i}
$$

Such combinations are very helpful (in particular, this makes it possible to consider rectangular random matrices). We shall use the notion of the dressed source matrices $C_{i}, i= \pm 1, \ldots, \pm n$ with the notation $\mathcal{L}_{X}[*]$ :

$$
\mathcal{L}_{X}\left[f\left(C_{1}, C_{-1} \ldots, C_{n}, C_{-n}\right)\right]:=f\left(X_{1} C_{1}, X_{-1} C_{-1} \ldots, X_{n} C_{n}, X_{-n} C_{-n}\right)
$$

where $f$ is any function. (The letter $\mathcal{L}$ is used to remember that it is the dressing from the left side.)

The matrices $\left\{C_{i}\right\}$ we call the source matrices which play the role of coupling constants in the matrix models below.

\subsection{Models Obtained from Graphs (Geometrical View)}

Consider a connected graph $\Gamma$ on an orientable connected surface $\Sigma$ without boundary with Euler characteristic E. We require such properties of the graph:

(1) its edges do not intersect. For example, the edges of the graph in Figure 1a do not intersect: the fact is that the graph is drawn on a torus, and not on a piece of paper.

(2) if we cut the surface $\Sigma$ along the edges of the graph, then the surface will decompose into disks (more precisely: into pieces homeomorphic to disks) (see Figure $3 \mathrm{a}, \mathrm{b}$ as an example).

As an example, see Figure 1, which contains all such graphs with two edges.

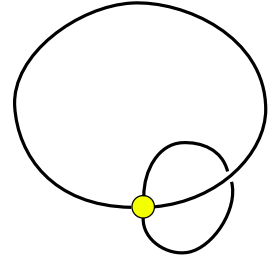

(a) 2 edges, 1 vertex, $\mathrm{E}=0$ (torus) Ex 2, Ex 9

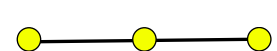

(b) 2 edges, 3 vertices, $\mathrm{E}=2$ (sphere) Ex 6, Ex 8 both

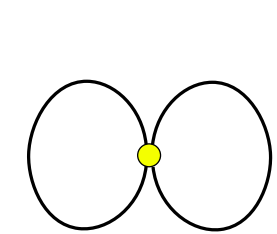

(c) 2 edges, 1 vertex, $\mathrm{E}=2$ (sphere) Ex 6, Ex 8 both

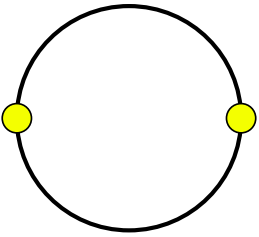

(d) 2 edges, 2 vertices, $\mathrm{E}=2$ (sphere) Ex 7

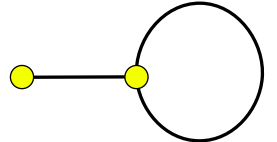

(e) 2 edges, 2 vertices, $\mathrm{E}=2$ (sphere) Ex 4

Figure 1. All possible graphs with 2 edges (two matrix models). Graph (b) is dual to (c). Ex means an example below. 
Such a graph is sometimes called a (clean) dessin d'enfants (the term dessin d'enfants without the additional "clean" serves for such a graph with a bipartite structure), sometimes-a map [28].

Let our graph have $\mathrm{F}$ faces, $n$ edges and $\mathrm{V}$ vertices; then $\mathrm{E}=\mathrm{V}-n+\mathrm{F}$.

We number all stars (i.e., all vertices of the graph $\Gamma$ ) with numbers from 1 to $V$ and all faces of $\Gamma$ with numbers from 1 to $\mathrm{F}$ and all edges of $\Gamma$ with numbers from 1 to $n$ in any way.

We will slightly expand the vertices of the graph and turn them into the small disk, which sometimes for the sake of visual clarity we will call stars.

In this case, the edges coming out of the vertex will divide the border of a small disk into segments. We orient the boundary of each such segment clockwise, and the segment can be represented by an arrow that goes from one edge to another; see the Figures $2 a, b, d$ and $3 a, c$ as examples. Our graph will have a total of $2 n$ arrow segments.

It is more correct to assume that the edges of the graph are very thin ribbons; that is, they have finite thickness. That is, the edge number $|i|$ has two sides, one of which we number with the number $i$, and the other with the number $-i$ (this choice is arbitrary but fixed). It would be more correct to depict each edge of $\Gamma$ numbered by $|i|$ in the form of a ribbon, the sides of which are two oppositely directed arrows; one arrow has a number $i$, and the second $-i$. However, with the exception of Figure 3 , we did not do that so as not to clutter up the drawings.

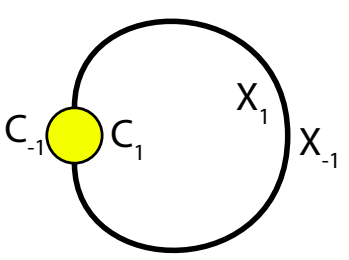

(a) 1 edges (1matrix model), 1 vertex (1 star), $\mathrm{E}=0$ (sphere)Ex 1 and Lemmas 3,5

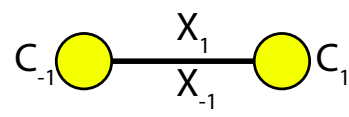

(b) 1 edges, 2 vertices, $\mathrm{E}=2$ (sphere) Ex 1 and Lemmas 2,4

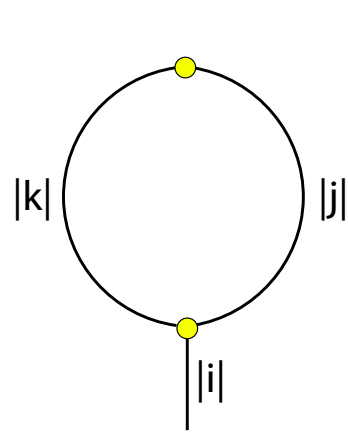

(c) A fragment of a graph with numbers on the edges

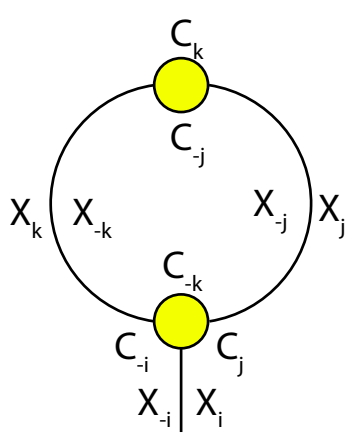

(d) The same fragment of a graph with random matrices on the edges and source matrices on stars

Figure 2. Decorated graphs.

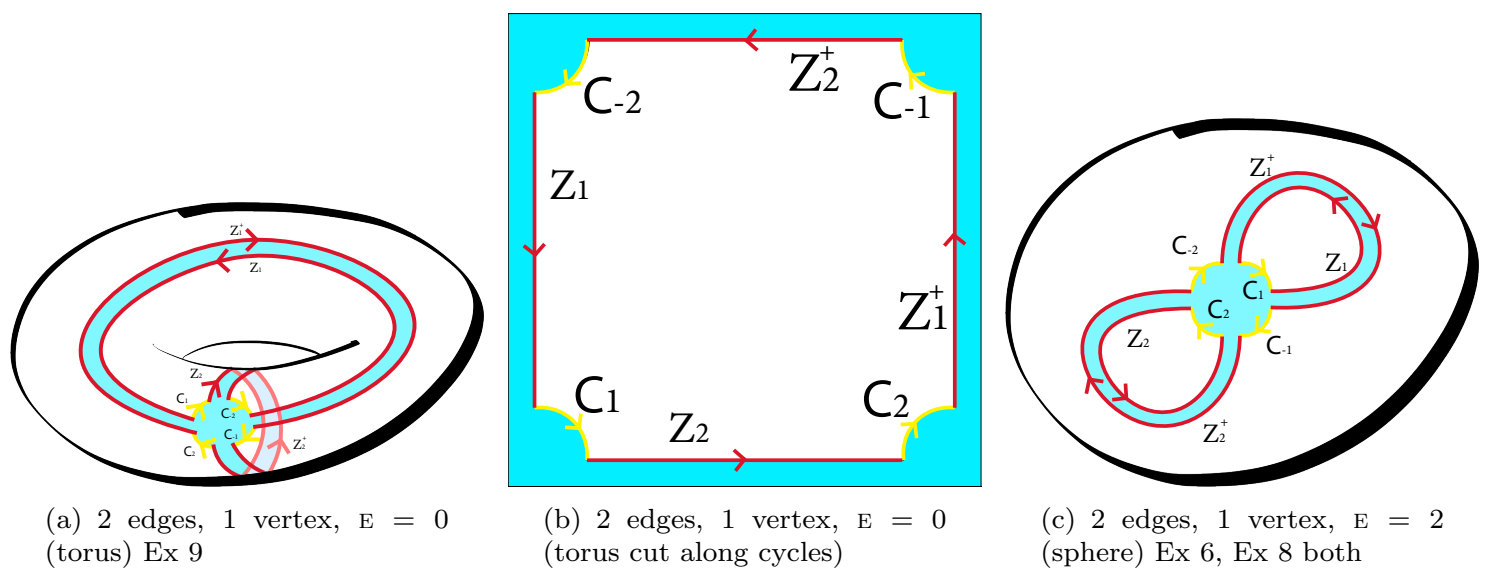

Figure 3. Graphs with 2 edges (two matrix models) where (a) is the zoom of (a) in Figure 1 and (c) is the zoom of (c) in Figure 1.

Now, each arrow of the side of the edge rests with its end against the arrow-segment-as if continued by the arrow-segment. We assign the same number $i(i= \pm 1, \ldots, \pm n)$ to the side of the 
edge $\Gamma$ and to the segment of the small disk, which continues this side while traversing the face in the positive direction.

Additionally, to the number $i(i= \pm 1, \ldots, \pm n)$ we attribute the product of the $N \times N$ matrices

$$
i \rightarrow X_{i} C_{i}
$$

where $C_{i}$ is assigned to the $i$ segment of a small disk and will be called source matrix, and $X_{i}$ is assigned to the arrow $i$, which is the side of the edge $|i|$ of the graph $\Gamma$; see Figure 2 for an illustration.

Let us use the following numbering. If one side of the edge of the tape $|i|$ is labeled $i$, then the other side of the same edges is labeled $-i$. (It does not matter which side of the edge $|i|$ we assign the number $i$ to, and which we assign the number $-i$ to, but we should fix the numbering we have chosen). The two sides of an edge of the graph $\Gamma$ are actually arrows pointing in opposite directions. (In order not to complicate the drawing, we do not depict these arrows in our figures, except for Figure $3 \mathrm{a}, \mathrm{b})$. We draw the arrows so that they point in the positive direction when bypassing the boundary of the face (that is, when bypassing the face in the counterclockwise direction). Each arrow, say $i$ (which either a positive or a negative number), ends at the boundary of the small disk (star) and with further bypassing of the face we pass along the segment of the boundary of the star. We assign the same number $i$ to this segment. Acting in this way, we give numbers to all segments of all small disks. It is easy to understand that all the arrows on the segments of the boundary of any small disc are directed clockwise (i.e., in the negative direction) when passing along the boundary of each small disk.

We attribute a sequential set of numbers to each star as follows. (this set is defined up to a cyclic permutation, and we will call it a cycle associated with the star). Examples: These are numbers $(1,-1)$ assigned to the star (yellow small disk) in Figure 2a. These are the numbers $(k,-j)$ that we attribute to the star on the top in the Figure $2 \mathrm{c}$ and the numbers $(-k, j,-i)$ that we attribute to the lower star in the same figure. Additionally, to each cycle we ascribe the related cyclic products:

$$
(1,-1) \leftrightarrow C_{1} C_{-1},
$$

to the star (yellow small disk) in Figure 2a. As for the stars in Figure 2c we obtain

$$
(k,-j) \leftrightarrow C_{k} C_{-j} \quad(-k, j,-i) \leftrightarrow C_{-k} C_{j} C_{-i}
$$

Each cycle product we will call the star's monodromy. As a result, a star's monodromy is a product of matrices that are attributed to arrow segments, taken in the same sequence in which arrows follow each other when moving around a small disk clockwise. We number the stars in any way by numbers from 1 to $\mathrm{V}$. The monodromy of a star $i$ will be denoted by the letter $W_{i}^{*}$.

In addition to the edges and in addition to the vertices, we number the faces of the graph $\Gamma$ and the corresponding face monodromies with numbers from 1 to $\mathrm{F}$. The cycles corresponding to the face $i$ will be denoted by $f_{i}$ and defined as follows. When going around the face border in the positive direction, namely, counterclockwise for an ordinary face (or counterclockwise if the face contains infinity), we collect segment numbers of small disks; for a face with a number $i$, this ordered collection of ordered numbers is $f_{i}$. As in the case of stars, we build cyclic products $f_{i} \leftrightarrow W_{i}$. Examples:

$$
f_{1}=(1) \leftrightarrow C_{1}=W_{1} \quad \text { and } \quad f_{2}=(-1) \leftrightarrow C_{-1}=W_{2}
$$

for two faces in Figure 2a.

We also introduce dressed monodromies:

$$
\mathcal{L}_{X}\left(W_{1}\right)=X_{1} C_{1}, \quad \mathcal{L}_{X}\left(W_{-1}\right)=X_{-1} C_{-1}
$$


Additionally, for the face in Figure 2c:

$$
f_{1}=(-j,-k) \leftrightarrow C_{-j} C_{-k}=W_{1} \quad \leftrightarrow \mathcal{L}_{X}\left(W_{1}\right)=X_{-j} C_{-j} X_{-k} C_{-k}
$$

Thus, we have two sets of cycles and two sets of monodromies: vertex cycles and vertex monodromies

$$
\sigma_{i}^{-1} \leftrightarrow W_{i}^{*}, \quad i=1, \ldots, \mathrm{V}
$$

and face cycles and face monodromies: The cycles corresponding to the face $i$ will be denoted by $f_{i}$; we have

$$
f_{i} \leftrightarrow W_{i} \leftrightarrow \mathcal{L}_{X}\left[W_{i}\right], \quad i=1, \ldots, \mathrm{F}
$$

Let us note that both cycles and monodromies are defined up to the cyclic permutation.

Remark 8. Important remark. Please note that each of the matrices $C_{i}, i= \pm 1, \ldots, \pm n$ enters the set of monodromies $W_{1}, \ldots, W_{f}$ once and only once. Accordingly, each random matrix $X_{i}, i= \pm 1, \ldots, \pm n$ is included once and only once in the set of dressed monodromies $\mathcal{L}_{X}\left[W_{1}\right], \ldots, \mathcal{L}_{X}\left[W_{f}\right]$. This determines the class of matrix models that we will consider and which we will call matrix models of dessin d'enfants.

Remark 9. In the description of maps (the same, of clean dessin d'enfants) the following combinatorial relation is well-known; see the wonderful texbook [28], Remark 1.3.19:

$$
\left(\prod_{i=1}^{n} \alpha_{i}\right)\left(\prod_{i=1}^{\mathrm{F}} f_{i}\right)=\left(\prod_{i=1}^{\mathrm{v}} \sigma_{i}^{-1}\right)
$$

where each of $\alpha_{i}, f_{i}$ and $\sigma_{i}$ belongs to the permutation group $S_{2 n}$. Here $f_{i}$ are face cycles, $\sigma_{i}$ are vertex cycles and $\left(\prod_{i=1}^{n} \alpha_{i}\right)$ is an involution without fixed points; each $\alpha_{i}$ transposes $i$ and $-i$. Since $\alpha_{i}^{2}=1$ we can also write

$$
\left(\prod_{i=1}^{n} \alpha_{i}\right)\left(\prod_{i=1}^{\mathrm{V}} \sigma_{i}^{-1}\right)=\left(\prod_{i=1}^{\mathrm{F}} f_{i}\right)
$$

The reader can check this relation for the pair of the simplest examples where $n=1$.

For any given set of the face cycles $f_{1}, \ldots, f_{\mathrm{F}}$, the relation (50) allows you to uniquely reconstruct the set of the vertex cycles $\sigma_{1}^{-1}, \ldots, \sigma_{V}^{-1}$ (the power -1 is related to the negative (clockwise) counting of numbers).

We get

\section{Proposition 1.}

$$
\hbar^{-n_{1}} \mathbb{E}_{n_{1}, n_{2}}\left\{\prod_{i=1}^{\mathrm{F}} \mathcal{L}_{X}\left[\operatorname{tr} W_{i}\right]\right\}=N^{-n_{2}} \prod_{i=1}^{\mathrm{V}} \operatorname{tr} W_{i}^{*}
$$

and

$$
\hbar^{-n_{1}} \mathbb{E}_{n_{1}, n_{2}}\left\{\prod_{i=1}^{\mathrm{v}} \mathcal{L}_{X}\left[\operatorname{tr} W_{i}^{*}\right]\right\}=N^{-n_{2}} \prod_{i=1}^{\mathrm{F}} \operatorname{tr} W_{i},
$$

where we use the notation (47) and where the parameters $n_{1}, n_{2}, \hbar$ were defined in Section 2.2.

We note that each trace is a sum of monomials. Let us note that each of $X_{i}, i= \pm 1, \ldots, \pm n$ enters only once in each monomials term insider of the integral in the left and side of (52) and of (53).

There are two ways to prove these relations: algebraic and geometrical ones. In short, the sketches of the proof are are as follows. We should notice that $\mathbf{p}_{(1)}(*)=s_{(1)}(*)$ and apply Lemmas $2-5$ while having in mind that this is actually the cut-or-join procedure (57) stated below in Section 3.3. In this case the difference between Lemmas 2, 3 and Lemmas 4, 5 is only in the power of the factor $N$, since $(N)_{\lambda}=N$ in case $\lambda=(1)$. 
Geometric way: We use the relation

$$
\hbar^{-1} \mathbb{E}_{n_{1}, 0}\left\{\left(Z_{i}\right)_{a, b}\left(Z_{j}^{\dagger}\right)_{b^{\prime}, a^{\prime}}\right\}=\delta_{i, j} \delta_{a, a^{\prime}} \delta_{b, b^{\prime}}
$$

and calculate monodromies along faces of the graph $\Gamma$ as described in the beginning of this section.

Remark 10. From geometrical point of view in this way we create a surface by gluing the polygons related to the dressed face monodromies; see [31].

Remark 11. You may notice some similarities between formulas (52), (53) and formulas (50), (51). Additionally, there is. One can say that the role of involution $\alpha_{i}$ is played by the integration over the matrix $X_{i}$ (by the Wick coupling of $X_{i}$ and $\left.X_{-i}\right)$.

We draw attention to two facts.

- $\quad$ The answer (i.e., the left-hand side of (52)) depends only on the spectrum of star monodromies

$$
\operatorname{Spect}\left(W_{1}^{*}\right), \ldots, \operatorname{Spect}\left(W_{\mathrm{V}}^{*}\right) \text {. }
$$

- The answer does not depend on how exactly in our model we distribute the matrices $\{Z\}$ and $\{U\}$ to dress the source matrices-only two numbers $n_{1}$ and $n_{2}$ are important. For example, it does not matter, in the right-hand side of (52), whether we dress $\mathcal{L}_{X}\left[C_{i}, C_{-i}\right]=U_{i} C_{i}, U_{-i} C_{-i}$ and $\mathcal{L}_{X}\left[C_{j}, C_{-j}\right]=Z_{j} C_{j}, Z_{-j} C_{-j}$ or $\mathcal{L}_{X}\left[C_{i}, C_{-i}\right]=Z_{i} C_{i}, Z_{-i} C_{-i}$ and $\mathcal{L}_{X}\left[C_{j}, C_{-j}\right]=U_{j} C_{j}, U_{-j} C_{-j}$.

\subsection{Our Models. Algebraic View}

You can forget about graphs and dessin d'enfants and set them out differently.

We have a set of random matrices

$$
X_{ \pm 1}, \ldots, X_{ \pm n}, \quad X_{-i}=X_{i}^{+}
$$

and there are as many source matrices

$$
C_{ \pm 1}, \ldots, C_{ \pm n}
$$

and we want to consider expectation value of the products of these matrices. We want each matrix $X_{i}$ to come in combination with the source matrix labeled with the same $i$; see (46) and (47).

The set (54) we will call the alphabet of pairs and the matrices $\left\{C_{i}\right\}$ can be considered letters of the alphabet.

The products of these matrices can be considered as words constructed from letters of the alphabet of pairs. Consider a group of words

$$
W_{1}, \ldots, W_{\mathrm{F}}
$$

with the following condition: each of matrices $C_{i}, i= \pm 1, \ldots, \pm n$ is included once and only once in one of the words of this group.

In addition, we ask the following property: In this group of words there is no such subset of words that could be constructed from the alphabet of pairs with a smaller set of pairs of letters (in other words, we will consider only connected groups of words. The connected group of words will be related to the connected graphs $\Gamma$ ).

Each word $W_{i}$ can be associated with an ordered set of numbers $f_{i}$ consisting of the numbers of the matrices included in the word: $f_{i} \leftrightarrow W_{i}$. Recall that each word $W_{i}$ (and respectively $f_{i}$ ) is defined up to a cyclic permutation.

There is a one-to-one correspondence between dessin d'enfants with $n$ edges and $\mathrm{F}$ faces and word sets constructed in this way. Based on the set $W_{1}, \ldots, W_{\mathrm{F}}$, the surface $\Sigma$ is built uniquely. To do this, the procedure is as follows. First, for each word, say $W_{i}$, we associate the polygon with the edges, 
numbered by the numbers of the matrices in the product, namely, by the set $f_{i}$. By going around the boundary of the polygon counterclockwise, we assign the numbers of the matrices to the edges if we write the word from left to right. We get a set of F polygons. After that, we glue these polygons so that the side with the number $i$ sticks together with the side with the number $-i$. We assign an orientation to each polygon, considering its edges arrows, showing the direction of counterclockwise. We glue the edges so that the beginning of the arrow $i$ sticks together with the end of the arrow $-i$. We get the surface $\Sigma$ and on it is the graph $\Gamma$, whose edges are glued from two oppositely directed arrows (ribbon edge).

To determine the Euler characteristic of $\Sigma$ we need to know the number of vertices of the graph $\Gamma$.

Cut-or-join procedure. Purely algebraically, one should act like this.

Consider $W_{1} \otimes W_{2} \otimes \cdots \otimes W_{\mathrm{F}}$ (the order in this tensor product is not important) and the set of involutions $T_{i}, i=1, \ldots, n$, which act on this tensor product as follows. Each involution of $T_{i}$ does not affect those $W_{a}$ that contain neither $C_{i}$ nor $C_{-i}$. Two situations are possible. (I) The matrices $C_{i}$ and $C_{-i}$ are in the same word, say, the word $W_{a}$. How then can we to rearrange the matrices with the word cyclically, to bring it to the form $C_{i} X C_{-i} Y$, where $X$ and $Y$ are some matrices. (II) The matrices $C_{i}$ and $C_{-i}$ are included in different words; in this case we will write these two words as $C_{i} X$ and $C_{-i} Y$. As you can see, the action of involutions $T_{i}$ corresponds to taking the integral in Lemmas 2-5. Then

$$
\begin{aligned}
& T_{i}\left[\cdots \otimes C_{i} X C_{-i} Y \otimes \cdots\right]=\cdots \otimes C_{i} X \otimes C_{i} Y \otimes \cdots \\
& T_{i}\left[\cdots \otimes C_{i} X \otimes C_{i} Y \otimes \cdots\right]=\cdots \otimes C_{i} X C_{-i} Y \otimes \cdots
\end{aligned}
$$

It is easy to see that involutions commute: $T_{i}\left[T_{j}[*]\right]=T_{j}\left[T_{i}[*]\right]$.

The transformation

$$
W_{1}, \ldots, W_{\mathrm{F}} \leftrightarrow W_{1}^{*}, \ldots, W_{\mathrm{V}}^{*}
$$

can be obtained purely algebraically in $n$ steps. We call these two sets of matrices dual sets.

Proposition:

$$
\begin{aligned}
& \prod_{i=1}^{n} T_{i}\left[W_{1} \otimes W_{2} \otimes \cdots \otimes W_{\mathrm{F}}\right]=W_{1}^{*} \otimes \cdots \otimes W_{\mathrm{V}}^{*} \\
& \prod_{i=1}^{n} T_{i}\left[W_{1}^{*} \otimes \cdots \otimes W_{\mathrm{V}}^{*}\right]=W_{1} \otimes W_{2} \otimes \cdots \otimes W_{\mathrm{F}}
\end{aligned}
$$

Remark 12. Actually this is a manifestation of the Equation (50) where $\alpha_{i}$ is related to $T_{i}$. An involution without fixed points $\prod_{i=1}^{n} T_{i}$ takes the graph $\Gamma$ to the graph dual to it.

Here are some examples (57) with explanations about the geometric representation in Figures 1-5 and in some other graphs:

Example 1. Suppose $W_{1}=C_{1}, W_{2}=C_{-1}$. In 1 step we get:

$$
W_{1} \otimes W_{2}=C_{1} \otimes C_{-1} \leftrightarrow C_{1} C_{-1}=W_{1}^{*}
$$

Therefore $\mathrm{V}=1$ and as we have $\mathrm{F}=2, n=1$; then the Euler characteristic is $\mathrm{E}=\mathrm{F}-n+\mathrm{V}=2$. The right hand side can be obtained from Figure $2 a$ as the face monodromies while the left-hand side can be obtained as star monodromies there. The left-hand side contains also the face monodromies in Figure $2 b$; and the right-hand side can be obtained as star monodromies there. (This is because graphs (a) and (b) are dual ones.)

Example 2. In 2 steps:

$$
W_{1}=C_{1} C_{2} C_{-1} C_{-2} \leftrightarrow C_{1} C_{-2} C_{-1} C_{2}=W_{1}^{*}
$$


Therefore $\mathrm{V}=1$, and as we have $\mathrm{F}=1, n=2$, then the Euler characteristic is $\mathrm{E}=\mathrm{F}-n+\mathrm{V}=0$ (torus). The right-hand side can be obtained from Figure $1 a$ as the face monodromies while the left-hand side can be obtained as star monodromies there; see the more detailed Figure 3a. The dual graph looks like the same as the graph on the torus: there is 1 vertex and 1 face; the left-hand side plays the role of the face monodromy for the dual graph. This a particular case of Ex 9 .

Example 3. In two steps:

$$
W_{1} \otimes W_{2} \otimes W_{3}=C_{-1} C_{-2} \otimes C_{1} \otimes C_{2} \leftrightarrow C_{1} C_{-1} C_{2} C_{-2}=W_{1}^{*}
$$

Therefore $\mathrm{V}=1$ and as we have $\mathrm{F}=3, n=2$, the Euler characteristic is $\mathrm{E}=\mathrm{F}-n+\mathrm{V}=2$. The right-hand side can be obtained from Figure $1 b$ as the face monodromies while the left-hand side can be obtained as star monodromies there. The left-hand side contains also the face monodromies in Figure $1 c$ (see Figure 3c for more details); the right-hand side can be obtained as star monodromies there. (This is because Figure $1 b$ and Figure $1 c$ are dual ones).

Example 4. In 2 steps:

$$
C_{1} C_{2} C_{-1} \otimes C_{-2} \leftrightarrow C_{1} C_{-2} C_{2} \otimes C_{-1}
$$

Therefore $\mathrm{V}=2$ and as we have $\mathrm{F}=2, n=2$, the Euler characteristic is $\mathrm{E}=\mathrm{F}-n+\mathrm{V}=2$. The right-hand side can be obtained from Figure 1e as the face monodromies while the left-hand side can be obtained as star monodromies there. The left-hand side contains also the face monodromies of the dual graph which is the graph of the same type (the loop from which the segment sticks out).

Example 5. In 5 steps:

$W_{1} \otimes W_{2} \otimes W_{3}=C_{1} C_{2} C_{3} C_{4} \otimes C_{-3} C_{-2} C_{5} \otimes C_{-5} C_{-1} C_{-4} \leftrightarrow C_{2} C_{-2} C_{-5} \otimes C_{2} C_{-3} \otimes C_{5} C_{3} C_{-4} \otimes C_{4} C_{-1}=W_{1}^{*} \otimes W_{2}^{*} \otimes W_{3}^{*} \otimes W_{4}^{*}$

Therefore $\mathrm{V}=4$ and as we have $\mathrm{F}=3, n=5$, the Euler characteristic is $\mathrm{E}=\mathrm{F}-n+\mathrm{V}=2$ (the same is obtained from Figure $5 d$ ).

Example 6. In $n$ steps:

$$
C_{1} C_{2} \cdots C_{n} C_{-n} \cdots C_{-1} \leftrightarrow C_{1} C_{-2} \otimes C_{2} C_{-3} \otimes \cdots \otimes C_{n-1} C_{-n} \otimes C_{n} \otimes C_{-1}
$$

Therefore $\mathrm{V}=n+1$ and as we have $\mathrm{F}=1$, the Euler characteristic is $\mathrm{E}=\mathrm{F}-n+\mathrm{V}=2$ The left-hand side is related to $\Gamma$ in form of a chain with $n+1$ vertices, $n$ edges drawn on the sphere (see Figure $1 \mathrm{~b}$ for the $n=2$ chain and see Figure 4 a for the $n=3$ chain). In case each source matrix is the identity ones, this is related to (44) which is a rather popular product. The right side is represented by such a graph: there are $n$ circles; each subsequent one decreases and is inside the previous one. They all touch at one point (the vertex); see Figure $4 b$.

Example 7. In $n$ steps:

$$
C_{1} C_{2} \cdots C_{n} \otimes C_{-n} C_{1-n} \cdots C_{-1} \leftrightarrow C_{1} C_{-2} \otimes C_{2} C_{-3} \otimes \cdots \otimes C_{n-1} C_{-n} \otimes C_{n} C_{-1}
$$

Therefore $\mathrm{V}=n$, and as we have $\mathrm{F}=2$, then the Euler characteristic is $\mathrm{E}=\mathrm{F}-n+\mathrm{V}=2$. (the case $n=2$ is obtained from Figure $1 d$ ). The left-hand side is related to $\Gamma$ in form of the chain from the previous case where we replace $n$ by $n-1$ and connect its ends by $n$-th edge. Namely, it is $n$-gon drawn on the sphere. The right-hand side is related to the graph where two vertices are connected by $n$ edges. In case $n=4$, the right-hand side can be obtained from Figure $4 d$ as the face monodromies while the left-hand side can be obtained as star monodromies there. The left-hand side contains also the face monodromies in Figure 4c; the right-hand side can be obtained as star monodromies there. (Indeed, graphs in Figure $4 d$ and in Figure $4 c$ are dual ones.) 
Example 8. In $n$ steps:

$$
C_{1} C_{-1} C_{2} C_{-2} \cdots C_{n} C_{-n} \leftrightarrow C_{-1} C_{-2} \cdots C_{-n} \otimes C_{1} \otimes C_{2} \otimes \cdots \otimes C_{n}
$$

Therefore $\mathrm{V}=n+1$, and as we have $\mathrm{F}=1$, then the Euler characteristic is $\mathrm{E}=\mathrm{F}-n+\mathrm{V}=2$. The left-hand side is related to a star-graph $\Gamma$; the right-hand side-to a petal graph (as an example take $n=3$ and look at Figure 5a and dual Figure $5 b$.

After the dressing procedure, in case all source matrices are chosen to be identical ones we get a popular product (43).

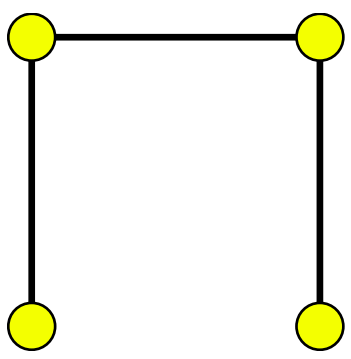

(a) 3 matrix model, $\mathrm{E}=2$ (sphere), Ex 6

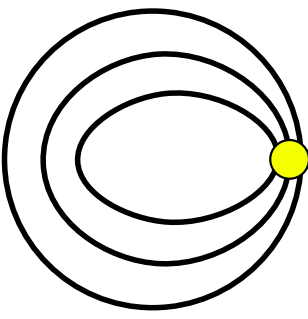

(b) 3 matrix model, $\mathrm{E}=2$ (sphere) Ex 6

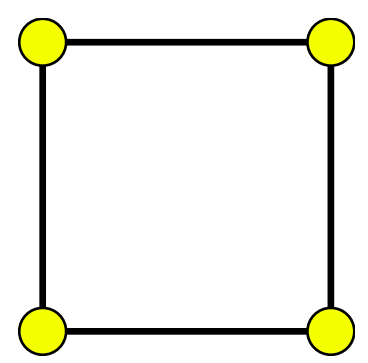

(c) 4 matrix model, $\mathrm{E}=2$ (sphere) Ex 7

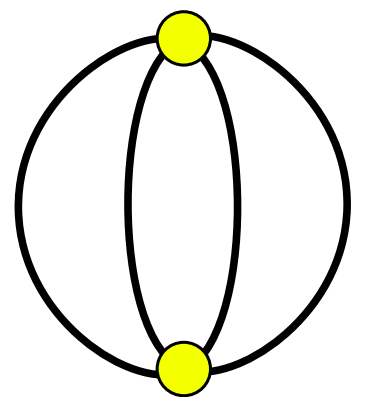

(d) 4 matrix model, $\mathrm{E}=2$ (sphere) Ex 7

Figure 4. Graphs drawn without decoration. Graph (a) is dual to (b). Graph (c) is dual to (d).

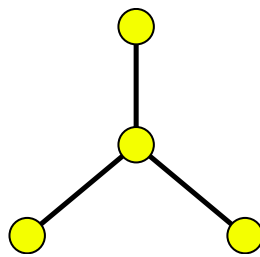

(a) 3 matrix model, $\mathrm{E}=2$ (sphere) Ex 8

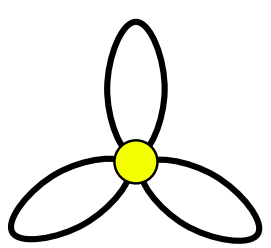

(b) 3 matrix model, $\mathrm{E}=2$ (sphere) Ex 8

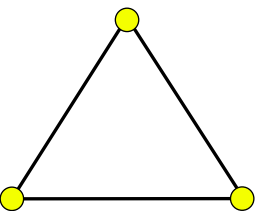

(c) 3 matrix model, $\mathrm{E}=2$ (sphere) Ex 7

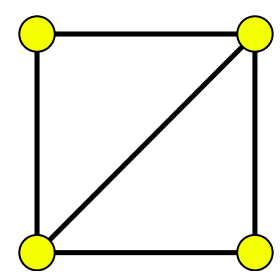

(d) 5 matrix model, $\mathrm{E}=2$ (sphere) Ex 5

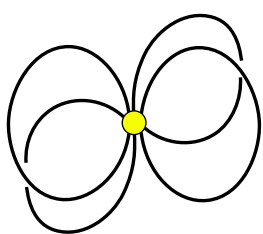

(e) 4 matrix model, $\mathrm{E}=-2$ (pretzel) Ex 9

Figure 5. Graphs drawn without decoration. Graph (a) is dual to (b).

Example 9. Suppose $W_{1}=C_{a_{1}} C_{b_{1}} C_{-a_{1}} C_{-b_{1}} \cdots C_{a_{g}} C_{b_{g}} C_{-a_{g}} C_{-b_{g}}$, where we number source matrices according $a_{i}, b_{i}$-cycles structure of a Riemann surface of genus $g$. In $n=2 g$ steps we obtain

$$
C_{a_{1}} C_{b_{1}} C_{-a_{1}} C_{-b_{1}} \cdots C_{a_{g}} C_{b_{g}} C_{-a_{g}} C_{-b_{g}} \leftrightarrow C_{-a_{1}} C_{b_{1}} C_{a_{1}} C_{-b_{1}} \cdots C_{-a_{g}} C_{b_{g}} C_{a_{g}} C_{-b_{g}}
$$

Therefore $\mathrm{V}=1$, and as we have $\mathrm{F}=1, n=2 g$, then the Euler characteristic is $\mathrm{E}=\mathrm{F}-n+\mathrm{V}=2-2 g$.

The case $n=2$ yields a torus and was considered in Example 2. The case $n=4$ can be related to a graph drawn on a pretzel; see Figure 5e. The left-hand side and the right-hand sides are related to dual graphs each of which has one face and one vertex and is drawn on a surface with genus $g$ whose edges are $a_{i}, b_{i}$ cycles.

Example 10. In $n=6$ steps we obtain

$$
C_{-1} C_{-2} C_{-3} \otimes C_{-5} C_{-4} C_{1} \otimes C_{-6} C_{5} C_{2} \otimes C_{6} C_{4} C_{3} \leftrightarrow C_{1} C_{4} C_{-3} \otimes C_{-5} C_{-1} C_{2} \otimes C_{-6} C_{-2} C_{3} \otimes C_{-4} C_{5} C_{6}
$$

Therefore $\mathrm{V}=4$, and as we have $\mathrm{F}=4, n=6$, then the Euler characteristic is $\mathrm{E}=\mathrm{F}-n+\mathrm{V}=2$. This can be represented as a tetrahedron inscribed in a sphere. The tetrahedron graph is self-dual. 


\section{Expectation Values of Matrix Products}

Lemmas 2-5 are generalized to the case of mixed ensembles; see Propositions 2 and 4 below.

Propositions $2-4$ are extended versions of statements studied in [31,32,37].

Proposition 2. Consider ensemble (29). Consider dual sets $W_{1}, \ldots, W_{\mathrm{F}}$ and $W_{1}^{*}, \ldots, W_{\mathrm{V}}^{*}$ of (57). For any given set of partitions $\lambda^{1}, \lambda^{2}, \ldots, \lambda^{\mathrm{F}}=\lambda \in \mathrm{Y}_{d}$, we have

$$
\mathbb{E}_{n_{1}, n_{2}}\left\{\mathcal{L}_{X}\left[s_{\lambda^{1}}\left(W_{1}\right) \cdots s_{\lambda^{\mathrm{F}}}\left(W_{\mathrm{F}}\right)\right]\right\}=\delta_{\lambda^{1}, \lambda^{2}, \ldots, \lambda^{\mathrm{F}}} \hbar^{n_{1} d}\left(s_{\lambda}\left(\mathbf{p}_{\infty}\right)\right)^{-n_{1}}\left(s_{\lambda}\left(\mathbb{I}_{N}\right)\right)^{-n_{2}} s_{\lambda}\left(W_{1}^{*}\right) \cdots s_{\lambda}\left(W_{\mathrm{V}}^{*}\right),
$$

where $\delta_{\lambda^{1}, \lambda^{2}, \ldots, \lambda^{\mathrm{F}}}$ is equal to 1 in case $\lambda^{1}=\lambda^{2}=\cdots=\lambda^{\mathrm{F}}$ and to 0 otherwise.

Similarly, for any set of partitions $\lambda^{1}, \lambda^{2}, \ldots, \lambda^{\mathrm{v}}=\lambda \in \mathrm{Y}_{d}$, we get

$$
\mathbb{E}_{n_{1}, n_{2}}\left\{\mathcal{L}_{X}\left[s_{\lambda^{1}}\left(W_{1}^{*}\right) \cdots s_{\lambda^{\mathrm{F}}}\left(W_{\mathrm{V}}^{*}\right)\right]\right\}=\delta_{\lambda^{1}, \lambda^{2}, \ldots, \lambda^{\vee}} \hbar^{n_{1} d}\left(s_{\lambda}\left(\mathbf{p}_{\infty}\right)\right)^{-n_{1}}\left(s_{\lambda}\left(\mathbb{I}_{N}\right)\right)^{-n_{2}} s_{\lambda}\left(W_{1}\right) \cdots s_{\lambda}\left(W_{\mathrm{F}}\right),
$$

where $\delta_{\lambda^{1}, \lambda^{2}, \ldots, \lambda^{\mathrm{F}}}$ is equal to 1 in case $\lambda^{1}=\lambda^{2}=\cdots=\lambda^{\mathrm{F}}$ and to 0 otherwise.

The sketch of proof. The proof is based on the cut-or-join procedure (57) of Section 3.3. which is the result of the step-by-step application of Lemmas 2-5. The different (geometrical) proof is based on the treating of the Wick rule as a way to glue surfaces from polygons and the use of (11) and of (27), (28).

Corollary 2. Consider ensemble (29). Consider dual sets $W_{1}, \ldots, W_{\mathrm{F}}$ and $W_{1}^{*}, \ldots, W_{\mathrm{V}}^{*}$ of (57), such that $\operatorname{det} W_{i}, \operatorname{det} W_{j}^{*} \neq 0$ for $i=1, \ldots, \mathrm{F}, j=1, \ldots, \mathrm{V}$. Suppose that $\alpha_{m}=\max \left(\alpha^{1}, \ldots, \alpha^{\mathrm{F}}\right)=: \alpha$, where $\alpha^{1}, \ldots, \alpha^{\mathrm{F}}$ is a given set of nonnegative integers. Consider a given set of partitions $\lambda^{i}, i=1, \ldots, \mathrm{F}$ and denote $\lambda=\lambda^{(m)}$. We have

$$
\begin{gathered}
\mathbb{E}_{n_{1}, n_{2}}\left\{\mathcal{L}_{X}\left[\prod_{i=1}^{\mathrm{F}} s_{\lambda^{i}}\left(W_{i}\right) \operatorname{det}\left(\left(W_{i}\right)^{\alpha_{i}}\right)\right]\right\}= \\
\delta_{\lambda^{1}+\alpha_{1}, \lambda^{2}+\alpha_{2}, \ldots, \lambda^{\mathrm{F}}+\alpha_{f}}\left(\hbar^{-(|\lambda|+\alpha N)} s_{\lambda}\left(\mathbf{p}_{\infty}\right) \frac{(N)_{\lambda}}{(N+\alpha)_{\lambda}}\right)^{-n_{1}}\left(s_{\lambda}\left(\mathbb{I}_{N}\right)\right)^{-n_{2}} \prod_{i=1}^{\mathrm{V}} s_{\lambda}\left(W_{i}^{*}\right) \operatorname{det}\left(\left(W_{i}^{*}\right)^{\alpha}\right)
\end{gathered}
$$

where $\delta_{\lambda^{1}+\alpha_{1}, \lambda^{2}+\alpha_{2}, \ldots, \lambda^{\mathrm{F}}+\alpha_{\mathrm{F}}}$ is equal to 1 in case for each $k=1, \ldots, N$ we have $\lambda_{k}^{(1)}+\alpha_{1}=\lambda_{k}^{(2)}+\alpha_{2}=\cdots=$ $\lambda_{k}^{(\mathrm{F})}+\alpha_{\mathrm{F}}$, and it is 0 otherwise.

Proposition 3 ([32]). let $\Delta^{i}=\left(\Delta_{1}^{i}, \Delta_{2}^{i}, \ldots\right), i=1, \ldots, k$ be a set of partitions of the weights $d_{1}, \ldots, d_{k}=d$. We get

Let $\mu^{k+1}=\left(\mu_{1}^{(k+1)}, \mu_{2}^{(k+1)}, \ldots\right), \ldots, \mu^{(\mathrm{F})}=\mu=\left(\mu_{1}, \mu_{2}, \ldots\right)$, where $1<k<\mathrm{F}$, be a set of partitions.

$$
\begin{gathered}
\mathbb{E}_{n_{1}, n_{2}}\left\{\mathcal{L}_{X}\left[\prod_{i=1}^{k} \mathbf{p}_{\Delta^{i}}\left(W_{i}\right) \prod_{i=k+1}^{\mathrm{F}} s_{\mu^{i}}\left(W_{i}\right)\right]\right\}= \\
\delta_{|\mu|, d} \delta_{d_{1}, \ldots, d_{k}} \delta_{\mu^{k+1}, \ldots, \mu^{\mathrm{F}-k}} \hbar^{n_{1} d}\left((N)_{\mu}\right)^{-n_{2}}\left(\frac{\operatorname{dim} \mu}{d !}\right)^{-n_{1}-n_{2}} \chi_{\mu}\left(\Delta^{1}\right) \cdots \chi_{\mu}\left(\Delta^{k}\right) \prod_{i=1}^{\mathrm{V}} s_{\lambda}\left(W_{i}^{*}\right)
\end{gathered}
$$

where $(N)_{\mu}$ is given by (40) and $\chi_{\mu}(\Delta)$ is the character of the symmetric group; see Remark (5). The symbol $\delta_{\mu^{k+1}, \ldots, \mu^{\mathrm{F}}}$ is equal to 1 in case $\mu^{k+1}=\cdots=\mu^{\mathrm{F}-k}$ and is equal to 0 otherwise.

Similarly, let $\mu^{k+1}, \ldots, \mu^{\mathrm{v}}=\mu$, where $1<k<\mathrm{v}$, be a set of partitions. Then

$$
\begin{gathered}
\mathbb{E}_{n_{1}, n_{2}}\left\{\mathcal{L}_{X}\left[\prod_{i=1}^{k} \mathbf{p}_{\Delta^{i}}\left(W_{i}^{*}\right) \prod_{i=k+1}^{\mathrm{v}} s_{\mu^{i}}\left(W_{i}^{*}\right)\right]\right\}= \\
\delta_{|\mu|, d} \delta_{d_{1}, \ldots, d_{k}} \delta_{\mu^{k+1}, \ldots, \mu^{v-k}} \hbar^{n_{1} d}\left((N)_{\mu}\right)^{-n_{2}}\left(\frac{\operatorname{dim} \mu}{d !}\right)^{-n_{1}-n_{2}} \chi_{\mu}\left(\Delta^{1}\right) \cdots \chi_{\mu}\left(\Delta^{k}\right) \prod_{i=1}^{\mathrm{F}} s_{\lambda}\left(W_{i}\right)
\end{gathered}
$$


The Proposition is derived from the previous one using (5) and (11).

Proposition 4. Let $\Delta^{i}=\left(\Delta_{1}^{i}, \Delta_{2}^{i}, \ldots\right), i=1, \ldots, \mathrm{F}$ be a set of partitions of weights $d_{1}, \ldots, d_{\mathrm{F}}=d$.

Then

$$
\begin{gathered}
\mathbb{E}_{n_{1}, n_{2}}\left\{\mathcal{L}_{X}\left[\mathbf{p}_{\Delta^{1}}\left(W_{1}\right) \cdots \mathbf{p}_{\Delta^{\mathrm{F}}}\left(W_{\mathrm{F}}\right)\right]\right\} \prod_{i=1}^{\mathrm{F}} \frac{1}{z_{\Delta^{i}}}= \\
\delta_{d_{1}, \ldots, d_{\mathrm{F}}} \hbar^{n_{1} d} \sum_{\widetilde{\Delta}^{1}, \ldots, \widetilde{\Delta}^{\mathrm{V}} \in \mathrm{Y}_{d}} \mathbf{p}_{\tilde{\Delta}^{1}}\left(W_{1}^{*}\right) \cdots \mathbf{p}_{\tilde{\Delta}^{\mathrm{V}}}\left(W_{\mathrm{V}}^{*}\right) H_{\mathrm{E}}\left(\widetilde{\Delta}^{1}, \ldots, \widetilde{\Delta}^{\mathrm{V}}, \Delta^{1}, \ldots, \Delta^{\mathrm{F}} \mid n_{2}\right),
\end{gathered}
$$

where $\delta_{d_{1}, \ldots, d_{\mathrm{F}}}=1$ in case $d_{1}=\cdots=d_{\mathrm{F}}$ and 0 otherwise, and $\mathrm{Y}_{d}$ is the set of all partitions of weight $d$. The prefactor $H_{\mathrm{E}}\left(\widetilde{\Delta}^{1}, \ldots, \widetilde{\Delta}^{\mathrm{V}}, \Delta^{1}, \ldots, \Delta^{\mathrm{F}} \mid n_{2}\right)$ in (69) is the weighted Hurwitz number (28) with $k=\mathrm{F}+\mathrm{V}$ and $\mathrm{E}=\mathrm{F}-n+\mathrm{V}$.

Similarly, for a given set of partitions $\tilde{\Delta}^{i}=\left(\tilde{\Delta}_{1}^{i}, \tilde{\Delta}_{2}^{i}, \ldots\right), i=1, \ldots, \mathrm{V}$ with weights $d_{1}, \ldots, d_{\mathrm{V}}=d$ respectively, we get

$$
\begin{gathered}
\mathbb{E}_{n_{1}, n_{2}}\left\{\mathcal{L}_{X}\left[\mathbf{p}_{\tilde{\Delta}^{1}}\left(W_{1}^{*}\right) \cdots \mathbf{p}_{\tilde{\Delta}^{\mathrm{v}}}\left(W_{\mathrm{V}}^{*}\right)\right]\right\}= \\
\delta_{d_{1}, \ldots, d_{\mathrm{V}}} \hbar^{n_{1} d} \sum_{\widetilde{\Delta}^{1}, \ldots, \widetilde{\Delta}^{\mathrm{F}} \in \mathrm{Y}_{d}} \mathbf{p}_{\Delta^{1}}\left(W_{1}\right) \cdots \mathbf{p}_{\Delta^{\mathrm{v}}}\left(W_{\mathrm{V}}\right) H_{\mathrm{E}}\left(\widetilde{\Delta}^{1}, \ldots, \widetilde{\Delta}^{\mathrm{V}}, \Delta^{1}, \ldots, \Delta^{\mathrm{F}} \mid n_{2}\right),
\end{gathered}
$$

where $H_{\mathrm{E}}\left(\widetilde{\Delta}^{1}, \ldots, \widetilde{\Delta}^{\mathrm{V}}, \Delta^{1}, \ldots, \Delta^{\mathrm{F}}\right)$ is exactly the same as in (69).

Propositions 2-4 and are equivalent. This can be proven with the help of (10), (11) and (28).

Remark 13. Proposition 4 was proved in [31] using a geometrical construction of Hurwitz numbers as a number of ways to glue polygons. Each matrix entry, say $\left(X_{i}\right)_{a, b}$, may be drawn as an arrow with labels a and $b$ at the startpoint and the endpoint respectively. We draw solid arrow for an entry of a random matrix and $a$ dashed arrow for an entry of a source matrix. The product of matrices we draw as arrows sequentially assigned to each other. The trace of a product is drawn as a polygon. Now each $L_{X}\left[t r W_{c}\right]$ is a polygon with alternating solid and dashed-edge arrows; we orient the edges counterclockwise. In [31] we named such polygons countries. Thus, we relate each dressed word to a country. It may be shown that the expectation

$$
\mathbb{E}_{n, 0}\left\{L_{X}\left[\operatorname{tr}\left(W_{1}\right) \cdots \operatorname{tr}\left(W_{1}\right)\right]\right\}
$$

may be viewed as the result of gluing of the net of countries into a surface, say $\Sigma$, and $\mathrm{E}=\mathrm{F}-n+\mathrm{V}$ being the Euler characteristic of this surface. This is a result of the Gaussian integration over matrices $Z_{i}$. Oppositely directed solid arrows (corresponding to $Z_{i}$ and $Z_{i}^{\dagger}$ ) form sides of ribbon edges. These edges end at a boundary of disks (inflated vertices) with dashed boundaries (source matrices are attached to the segments of dashed boundaries). In [31] we named this disk watchtowers. There are $n$ ribbon edges (the boarders of countries) and $2 n$ dashed edges (segments of boundaries of disks—of the boarders of the watchtowers); there are $\mathrm{V}$ watchtowers; and there are $\mathrm{F}$ countries with alternating (solid-dashed) edges. There are $2 n$ 3-valent vertices (ends of ribbons): there are two dashed arrows (one is outgoing; another is incoming) and one ribbon (one side is the solid outgoing arrow; the other side is a incoming solid arrow) attached to each vertex. This is a graph $\Gamma$ drawn on $\Sigma$. This graph is related to

$$
\mathbb{E}_{n, 0}\left\{L_{X}\left[\operatorname{tr}\left(W_{1}\right) \cdots \operatorname{tr}\left(W_{1}\right)\right]\right\}=\hbar^{n} \operatorname{tr}\left(W_{1}^{*}\right) \cdots \operatorname{tr}\left(W_{\mathrm{V}}^{*}\right),
$$

which is (68) for $d=1$ (in this case, all $\Delta^{i}$ has weight 1 , and $p_{(1)}(W)=\operatorname{tr}(W)$ ). In case $d>1$, instead of each $\operatorname{tr}\left(W_{i}\right)$ we have the product

$$
\operatorname{tr}\left(\left(W_{i}\right)^{\Delta_{1}^{i}}\right) \cdots \operatorname{tr}\left(\left(W_{i}\right)^{\Delta_{\ell_{i}}^{i}}\right) \rightarrow \operatorname{tr}\left(W_{i}\right)
$$


One may interpret it as a projection of $\ell_{i}$ polygons to the country (the polygon) labeled by $i$.

Remark 14. Notice that the answers for the expectation values which were considered above depend only on eigenvalues of $W_{i}^{*}$ or $W_{i} i=1, \ldots, \mathrm{v}$.

\section{Examples of Matrix Models}

Recall that in Section 2.1 we introduced the function $\tau_{r}\left(n, \mathbf{p}^{(1)}, \mathbf{p}^{(2)}\right)$. In what follows we use the conventions:

$$
\tau_{r}\left(\mathbf{p}^{(1)}, \mathbf{p}^{(2)}\right):=\tau_{r}\left(0, \mathbf{p}^{(1)}, \mathbf{p}^{(2)}\right)=\sum_{\lambda} r_{\lambda} s_{\lambda}\left(\mathbf{p}^{(1)}\right) s_{\lambda}\left(\mathbf{p}^{(2)}\right), \quad r_{\lambda}=r_{\lambda}(0)
$$

It depends on two sets $\mathbf{p}^{i}=\left(p_{1}^{(i)}, p_{2}^{(i)}, \ldots\right), i=1,2$, and on the choice of an arbitrary function of the variable $r$. (This is an example of the so-called tau function, but we will not use this fact.) As one of their sets, we will choose $\mathbf{p}^{2}=\mathbf{p}(X)$ like in (21), and the second set will be the set of arbitrary parameters. With $r=1$ we get

$$
\tau_{1}(\mathbf{p}, X)=e^{\sum_{m>0} \frac{1}{m} p_{m} \operatorname{tr}\left(X^{m}\right)}
$$

For example, if we take

$$
r(x)=\frac{\prod_{i}^{p}\left(a_{i}+x\right)}{\prod_{i}^{q}\left(b_{i}+x\right)}
$$

and in addition to $\mathbf{p}^{1}=(1,0,0, \ldots)$ we get the so-called hypergeometric function of the matrix argument:

$$
{ }_{p} F_{q}\left(\begin{array}{l}
a_{1}, \ldots, a_{p} \\
b_{1}, \ldots, b_{q}
\end{array} \mid X\right)=\sum_{\lambda} \frac{\operatorname{dim} \lambda}{|\lambda| !} s_{\lambda}(X) \frac{\prod_{i}^{p}\left(a_{i}+x\right)_{\lambda}}{\prod_{i}^{q}\left(b_{i}+x\right)_{\lambda}}
$$

Special cases:

$$
\begin{gathered}
e^{\operatorname{tr} X}=\sum_{\lambda} s_{\lambda}(X) \frac{\operatorname{dim} \lambda}{|\lambda| !} \\
\operatorname{det}(1-z X)^{-a}=\sum_{\lambda} z^{|\lambda|}(a)_{\lambda} s_{\lambda}(X) \frac{\operatorname{dim} \lambda}{|\lambda| !}
\end{gathered}
$$

Integrals. Using Proposition 2 we obtain

Theorem 1. Suppose $W_{1}, \ldots, W_{\mathrm{F}}$ and $W_{1}^{*}, \ldots, W_{\mathrm{V}}^{*}$ are dual sets $(57)$. Let sets $\mathbf{p}^{i}=\left(p_{1}^{(i)}, p_{2}^{(i)}, p_{3}^{(i)}, \ldots\right)$, $i=1, \ldots, \max (\mathrm{F}, \mathrm{V})$ be independent complex parameters and $r(i), i=1, \ldots, \max (\mathrm{F}, \mathrm{V})$ be a set of given functions in one variable.

$$
\begin{aligned}
& \mathbb{E}_{n_{1}, n_{2}}\left\{\mathcal{L}_{X}\left[\tau_{r^{(1)}}\left(\mathbf{p}^{1}, W_{1}\right) \cdots \tau_{r^{(\mathrm{F})}}\left(\mathbf{p}^{\mathrm{F}}, W_{\mathrm{F}}\right)\right]\right\} \\
& =\sum_{\lambda} r_{\lambda} \hbar^{n_{1}|\lambda|}\left(\frac{\operatorname{dim} \lambda}{|\lambda| !}\right)^{-n} \prod_{i=1}^{\mathrm{V}} s_{\lambda}\left(W_{i}^{*}\right) \prod_{i=1}^{\mathrm{F}} s_{\lambda}\left(\mathbf{p}^{i}\right),
\end{aligned}
$$

where each $\tau_{r^{(i)}}\left(\mathbf{p}^{i}, W_{i}\right)$ is defined by (21)

$$
r_{\lambda}=\left((N)_{\lambda}\right)^{-n_{2}} \prod_{i=1}^{\mathrm{F}} r_{\lambda}^{(i)}(n)
$$

Similarly

$$
\begin{aligned}
& \mathbb{E}_{n_{1}, n_{2}}\left\{\mathcal{L}_{X}\left[\tau_{r^{(1)}}\left(\mathbf{p}^{1}, W_{1}^{*}\right) \cdots \tau_{r^{(\mathrm{v})}}\left(\mathbf{p}^{\mathrm{v}}, W_{\mathrm{v}}^{*}\right)\right]\right\} \\
& =\sum_{\lambda} r_{\lambda} \hbar^{n_{1}|\lambda|}\left(\frac{\operatorname{dim} \lambda}{|\lambda| !}\right)^{-n} \prod_{i=1}^{\mathrm{F}} s_{\lambda}\left(W_{i}\right) \prod_{i=1}^{\mathrm{V}} s_{\lambda}\left(\mathbf{p}^{i}\right),
\end{aligned}
$$


Remark 15. We recall the convention (73) In (79) $r_{\lambda}$ is the content product (16)

$$
r_{\lambda}=r_{\lambda}(0)=\prod_{(i, j) \in \lambda} r(j-i)
$$

where

$$
r(x)=(N+x)^{-n_{2}} \prod_{i=1}^{\mathrm{F}} r^{(i)}(x)
$$

To get examples we choose

- $\quad$ Dual sets $W_{1}, \ldots, W_{\mathrm{F}} \leftrightarrow W_{1}^{*}, \ldots, W_{\mathrm{V}}^{*}$;

- The fraction of unitary matrices given by $n_{2}$;

- The set of functions $r^{(i)}, i=1, \ldots, \mathrm{F}$;

- $\quad$ The sets $\mathbf{p}^{(i)}, i=1, \ldots, \mathrm{F}$.

Remark 16. Answers in some cases are further simplified. Let us mark two cases

(i) Firstly, this is the case when the spectrum of the stars has the form

$$
\text { Spect } W_{i}^{*}=\operatorname{Spect} \mathbb{I}_{N, k_{i}}=\operatorname{diag}\{1,1, \ldots, 1,0,0, \ldots, 0\}, \quad i=1, \ldots, \mathrm{V}
$$

where $\mathbb{I}_{N, k_{i}}$ is the matrix with $k_{i}$ units of the main diagonal. Such star monodromies obtained in case source matrices have a rank smaller than $N$. Insertion of such matrices in the left-hand sides of (79) and (81) corresponds to the integration over rectangular random matrices. One should take into account that

$$
s_{\lambda}\left(\mathbb{I}_{N, k}\right)=(k)_{\lambda} s_{\lambda}\left(\mathbf{p}_{\infty}\right),
$$

where we recall the notation

$$
(a)_{\lambda}:=(a)_{\lambda_{1}}(a-1)_{\lambda_{2}} \cdots(a-\ell+1)_{\lambda_{\ell^{\prime}}}
$$

(ii) The case is the specification of the sets $\mathbf{p}^{i}, i=1, \ldots, \mathrm{F}$ according to the following

Lemma 6. Denote

$$
\begin{gathered}
\mathbf{p}_{\infty}=(1,0,0, \ldots) \\
\mathbf{p}(a)=(a, a, a, \ldots) \\
\mathbf{p}(q, t)=\left(p_{1}(q, t), p_{2}(q, t), \ldots\right), \quad p_{m}(q, t)=\frac{1-q^{m}}{1-t^{m}}
\end{gathered}
$$

Then

$$
\frac{s_{\lambda}(\mathbf{p}(a))}{s_{\lambda}\left(\mathbf{p}_{\infty}\right)}=(a)_{\lambda}, \quad \mathbf{p}(a)=(a, a, a, \ldots)
$$

where $(a)_{\lambda}:=(a)_{\lambda_{1}}(a-1)_{\lambda_{2}} \cdots(a-\ell+1)_{\lambda_{\ell^{\prime}}}(a)_{n}:=a(a+1) \cdots(a+n-1)$, where $\lambda=\left(\lambda_{1}, \ldots, \lambda_{\ell}\right)$ is a partition. More generally

$$
\frac{s_{\lambda}(\mathbf{p}(q, t))}{s_{\lambda}(\mathbf{p}(0, t))}=(q ; t)_{\lambda}
$$

where $(q ; t)_{\lambda}=(q ; t)_{\lambda_{1}}\left(q t^{-1} ; t\right)_{\lambda_{2}} \cdots\left(q t^{1-\ell} ; t\right)_{\lambda_{\ell}}$ where $(q ; t)_{k}=(1-q)(1-q t) \cdots\left(1-q t^{n-1}\right)$ is $t$-deformed Pochhammer symbol. $(q ; t)_{0}=1$ is implied.

For such specifications the right-hand side of (79) can ta

With such specifications, one can diminish the number of the Schur functions in the right-hand side of (79) (or of (81)) and the right-hand side can take one of the forms:

$$
\sum_{\lambda} r_{\lambda} s_{\lambda}(A) s_{\lambda}(B)
$$




$$
\begin{gathered}
\sum_{\lambda} r_{\lambda} s_{\lambda}(A) \\
\sum_{\lambda} r_{\lambda}
\end{gathered}
$$

For (89) there is a determinant representation; for (90) there is a Pfaffian representation and (91) can be rewritten as a sum of products. Indeed if we introduce $r(x)=e^{T_{x-1}-T_{x}}$, and $\lambda=\left(\lambda_{1}, \ldots, \lambda_{N}\right)$, then

$$
r_{\lambda}(m)=\prod_{(i, j) \in \lambda} r(m+j-i)=e^{T_{m}+\cdots+T_{m-N}} \prod_{i=1}^{N} e^{T_{\lambda_{i}-i+m}}
$$

For instance, one can take $r(x)=a+x$ and get

$$
(a)_{\lambda}=\frac{\Gamma\left(a+\lambda_{1}-1\right) \Gamma\left(a+\lambda_{2}-2\right) \cdots \Gamma\left(a+\lambda_{N}-N\right)}{\Gamma(a) \Gamma(a-1) \cdots \Gamma(a-N+1)}
$$

Then we introduce $h_{i}=\lambda_{i}-i+N$ and write

$$
\sum_{\lambda} \frac{(a)_{\lambda}}{(b)_{\lambda}}=\sum_{h_{1}>\cdots>h_{N} \geq 0} \prod_{i=1}^{N} \frac{\Gamma(b-i+1)}{\Gamma(a-i+1)} \frac{\Gamma\left(h_{i}+a-N\right)}{\Gamma\left(h_{i}+b-N\right)}
$$

where $\Gamma$ is the gamma-function. (In case the argument of gamma-function turns out to be a nonpositive integer one should keep in mind both the enumerator and denominator.) See examples below.

Example 11. See Example 1 and Figure 2a. Take $X_{1}=Z$ and $r$ given by (13). The example of (79) can be chosen as follows

$$
\begin{gathered}
\mathbb{E}_{1,0}\left\{{ }_{p} F_{q}\left(\begin{array}{c}
a_{1}, \ldots, a_{p} \\
b_{1}, \ldots, b_{q}
\end{array} \mid Z C_{1}\right){ }_{p^{\prime}} F_{q^{\prime}}\left(\begin{array}{c}
a_{1}^{\prime}, \ldots, a_{p^{\prime}}^{\prime} \\
b_{1}^{\prime}, \ldots, b_{q^{\prime}}^{\prime}
\end{array} \mid Z^{\dagger} C_{-1}\right) \operatorname{det}\left(Z Z^{\dagger}\right)^{\alpha}\right\}= \\
p^{\prime} F_{q^{\prime}}\left(\begin{array}{c}
a_{1}, \ldots, a_{p}, a_{1}^{\prime}, \ldots, a_{p^{\prime}}^{\prime} N+\alpha \\
b_{1}, \ldots, b_{q}, b_{1}^{\prime}, \ldots, b_{q^{\prime}}^{\prime}, N
\end{array} \mid C_{1} C_{-1}\right),
\end{gathered}
$$

corresponding determinantal representation see a (21).

See and Figure $2 b$ which is dual to Figure $2 a$. An example of (81) can be chosen as

$$
\begin{gathered}
\mathbb{E}_{1,0}\left\{p_{p^{\prime}} F_{q^{\prime}}\left(\begin{array}{c}
a_{1}, \ldots, a_{p}, a_{1}^{\prime}, \ldots, a_{p^{\prime}}^{\prime}, N+\alpha \\
b_{1}, \ldots, b_{q}, b_{1}^{\prime}, \ldots, b_{q^{\prime}}^{\prime}, N
\end{array} \mid Z C_{1} Z^{\dagger} C_{-1}\right) \operatorname{det}\left(Z Z^{+}\right)^{\beta}\right\}= \\
\sum_{\lambda} s_{\lambda}\left(C_{1}\right)_{\lambda}\left(C_{-1}\right) \frac{\left.(N+\alpha)_{\lambda}(N+\beta)_{\lambda}\right)}{\left((N)_{\lambda}\right)^{2}} \frac{\prod_{i}^{p}\left(a_{i}\right)_{\lambda}}{\prod_{i}^{q}\left(b_{i}\right)_{\lambda}} \frac{\prod_{i}^{p^{\prime}}\left(a_{i}\right)_{\lambda}}{\prod_{i}^{q^{\prime}}\left(b_{i}\right)_{\lambda}},
\end{gathered}
$$

The determinantal representation of the left-hand side is given by (15).

Example 12. See Example 2 and Figure $3 a$. Take $X_{1}=U_{1}, X_{2}=U_{2}$.

$$
\begin{gathered}
\mathbb{E}_{0,2}\left\{e^{\sum_{m>0} \frac{1}{m} p_{m} \operatorname{tr}\left(U_{1} C_{1} U_{2} C_{2} U_{1}^{\dagger} C_{-1} U_{2}^{\dagger} C_{-2}\right)^{m}}\right\} \\
=\sum_{\lambda} \frac{1}{\left((N)_{\lambda}\right)^{2}} \frac{s_{\lambda}(\mathbf{p}) s_{\lambda}\left(C_{1} C_{-2} C_{-1} C_{2}\right)}{\left(s_{\lambda}\left(\mathbf{p}_{\infty}\right)\right)^{2}}
\end{gathered}
$$


Let us take $\mathbf{p}=\left(a z, a z^{2}, a z^{3}, \ldots\right)$ and $W_{1}^{*}=\mathbb{I}_{N, k} ;$ see (85) and (82). We obtain the left-hand side as

$$
\begin{gathered}
\mathbb{E}_{0,2}\left\{\operatorname{det}\left(1-z U_{1} C_{1} U_{2} C_{2} U_{1}^{\dagger} C_{-1} U_{2}^{\dagger} C_{-2}\right)^{-a}\right\}=\sum_{\lambda} z^{|\lambda|} \frac{(a)_{\lambda}(k)_{\lambda}}{\left((N)_{\lambda}\right)^{2}}= \\
z^{-\frac{1}{2} N(N-1)} \sum_{h_{1}>\cdots>h_{N} \geq 0} \prod_{i=1}^{N} z^{h_{i}} \frac{(\Gamma(N-i+1))^{2}}{\Gamma(a-i+1) \Gamma(k-i+1)} \frac{\Gamma\left(h_{i}+a-N\right) \Gamma\left(h_{i}+k-N\right)}{\left(\Gamma\left(h_{i}\right)\right)^{2}}
\end{gathered}
$$

where Spect $C_{1} C_{-2} C_{-1} C_{2}=$ Spect $\mathbb{I}_{N, k}$ see (94).

Example 13. See Example 3 and Figure 3c.

$$
\begin{gathered}
\left.\mathbb{E}_{2,0}\left\{e^{\sum_{m>0} \frac{1}{m} p_{m}^{(1)} \operatorname{tr}\left(Z_{1} C_{1}\right)^{m}}+\sum_{m>0} \frac{1}{m} p_{m}^{(2)} \operatorname{tr}\left(Z_{2} C_{2}\right)^{m}\right) \operatorname{det}\left(1-z Z_{1}^{\dagger} C_{-1} Z_{2}^{\dagger} C_{-2}\right)^{-a} \prod_{i=1}^{3} \operatorname{det}\left(Z_{i} Z_{i}^{\dagger}\right)^{\alpha}\right\} \\
=\sum_{\lambda} z^{|\lambda|} s_{\lambda}\left(\mathbf{p}^{1}\right) s_{\lambda}\left(\mathbf{p}^{2}\right)(a)_{\lambda}\left(\frac{(N+\alpha)_{\lambda}}{(N)_{\lambda}}\right)^{3}
\end{gathered}
$$

For a determinant representation see (20)

Example 14. For decoration of Figure 1e we put $X_{i}=Z_{i}$.

$$
\mathbb{E}_{2,0}\left\{\mathbf{p}_{\Delta}\left(Z_{1}^{\dagger} C_{-1}\right) s_{\lambda}\left(Z_{1} C_{1} Z_{2} C_{2} Z_{2}^{\dagger} C_{-2}\right)\right\}=\delta_{|\lambda|,|\Delta|} \frac{\operatorname{dim} \lambda}{|\lambda| !} \chi_{\lambda}(\Delta) s_{\lambda}\left(C_{2}\right) s_{\lambda}\left(C_{1} C_{-2} C_{-1}\right)
$$

see (66).

Example 15. Figure $5 d$ in particular yields

$$
\begin{gathered}
\mathbb{E}_{2,0}\left\{s_{\lambda}\left(Z_{1} C_{1} Z_{2} C_{2} Z_{3} C_{3} Z_{4} C_{4}\right) s_{\lambda}\left(Z_{3}^{\dagger} C_{-3} Z_{2}^{\dagger} C_{-2} Z_{5} C_{5}\right) s_{\lambda}\left(Z_{5}^{\dagger} C_{-5} Z_{1}^{\dagger} C_{-1} Z_{4}^{\dagger} C_{-4}\right)\right\}= \\
\left(\frac{\operatorname{dim} \lambda}{|\lambda| !}\right)^{-5} s_{\lambda}\left(C_{1} C_{-2} C_{-5}\right) s_{\lambda}\left(C_{2} C_{-3}\right) s_{\lambda}\left(C_{5} C_{3} C_{-4}\right) s_{\lambda}\left(C_{4} C_{-1}\right) .
\end{gathered}
$$

Example 16. In the case below we use an open chain with $n$ edges as in Figures $1 b, 2 b$ and $4 a$.

$$
\mathbb{E}_{n, 0}\left\{e^{\sum_{m>0} \frac{1}{m} p_{m}^{(1)} \operatorname{tr}\left(\left(Z_{1} C_{1} Z_{2} C_{2} \cdots Z_{n} C_{n} Z_{n}^{\dagger} C_{-n} \cdots Z_{1}^{\dagger} C_{-1}\right)^{m}\right)}\right\}=\sum_{\lambda} s_{\lambda}\left(C_{n}\right) s_{\lambda}\left(C_{-1}\right) \frac{s_{\lambda}(\mathbf{p})}{s_{\lambda}\left(\mathbf{p}_{\infty}\right)} \prod_{i=1}^{n-1} \frac{s_{\lambda}\left(C_{i} C_{-i-1}\right)}{s_{\lambda}\left(\mathbf{p}_{\infty}\right)}
$$

Graphs dual to the chain look like in Figure $4 b$.

$$
\begin{gathered}
\mathbb{E}_{n, 0}\left\{e^{\sum_{m>0} \frac{1}{m} p_{m} \operatorname{tr}\left(\left(Z_{1}^{\dagger} C_{-1}\right)^{m}\right)+\sum_{m>0} \frac{1}{m} p_{m}^{(n)} \operatorname{tr}\left(\left(Z_{n} C_{n}\right)^{m}\right)} \prod_{i=1}^{n-1} e^{\sum_{m>0} \frac{1}{m} p_{m}^{(i)} \operatorname{tr}\left(\left(Z_{i} C_{i} Z_{i+1}^{\dagger} C_{-i-1}\right)^{m}\right)}\right\} \\
=\sum_{\lambda} s_{\lambda}(\mathbf{p}) s_{\lambda}\left(C_{1} C_{2} \cdots C_{n} C_{-n} \cdots C_{-1}\right) \prod_{i=1}^{n} \frac{s_{\lambda}\left(\mathbf{p}^{i}\right)}{s_{\lambda}\left(\mathbf{p}_{\infty}\right)}
\end{gathered}
$$

These relations generalize product (44) and (45).

Example 17. Our graph is a polygon with $n$ edges and $n$ vertices (stars); see Figures $1 d, 2 a$ and $4 c$ for examples.

$$
\mathbb{E}_{n, 0}\left\{e^{\sum_{m>0} \frac{1}{m} p_{m}^{(1)} \operatorname{tr}\left(Z_{1} C_{1} Z_{2} C_{2} \cdots Z_{n} C_{n}\right)^{m}+\sum_{m>0} \frac{1}{m} p_{m}^{(2)} \operatorname{tr}\left(Z_{n}^{+} C_{-n} Z_{n-1}^{+} C_{1-n} \cdots Z_{1}^{\dagger} C_{-1}\right)^{m}} \prod_{i=1}^{n} \operatorname{det}\left(Z_{i} Z_{i}^{\dagger}\right)^{\alpha}\right\}
$$




$$
=\sum_{\lambda} s_{\lambda}\left(\mathbf{p}^{1}\right) s_{\lambda}\left(\mathbf{p}^{2}\right) \prod_{i=1}^{n} \frac{(N+\alpha)_{\lambda} s_{\lambda}\left(C_{i} C_{-i-1}\right)}{(N)_{\lambda} s_{\lambda}\left(\mathbf{p}_{\infty}\right)}
$$

(where we put $C_{-n-1}=C_{-1}$ ).

A graph dual to the polygon can be viewed as two-stars graph with $n$ edges which connect stars; see Figures $1 d$ and $4 d$ as examples.

$$
\mathbb{E}_{n, 0}\left\{\prod_{i=1}^{n} e^{\sum_{m>0} \frac{1}{m} p_{m}^{(i)} \operatorname{tr}\left(Z_{i} C_{i} Z_{i+1}^{\dagger} C_{-i-1}\right)^{m}}\right\}=\sum_{\lambda} s_{\lambda}\left(C_{1} C_{2} \cdots C_{n}\right) s_{\lambda}\left(C_{-n} C_{1-n} \cdots C_{-1}\right) \prod_{i=1}^{n} \frac{s_{\lambda}\left(\mathbf{p}^{i}\right)}{s_{\lambda}\left(\mathbf{p}_{\infty}\right)}
$$

To apply determinantal formulas one should use Remark 16.

Example 18. Consider the star-graph with n-rays which end at other stars (see Figure $5 a$ where $n=3$ ). This situation corresponds to (43).

$$
\begin{gathered}
\mathbb{E}_{n, 0}\left\{e^{\sum_{m>0} \frac{1}{m} p_{m} \operatorname{tr}\left(Z_{1} C_{1} Z_{1}^{\dagger} C_{-1} \cdots Z_{n} C_{n} Z_{n}^{\dagger} C_{-n}\right)} \prod_{i=1}^{n} \operatorname{det}\left(Z_{i} Z_{i}^{\dagger}\right)^{\alpha}\right\}= \\
\sum_{\lambda} s_{\lambda}(\mathbf{p}) s_{\lambda}\left(C_{-1} C_{-2} \cdots C_{-n}\right) \prod_{i=1}^{n} \frac{(N+\alpha)_{\lambda} s_{\lambda}\left(C_{i}\right)}{(N)_{\lambda} s_{\lambda}\left(\mathbf{p}_{\infty}\right)}
\end{gathered}
$$

A similar model was studied in [16,22]. It has the determinantal representation (21) in case all $W_{i}^{*}$ except one are of form (82). There is the determinantal representation (15) in case we specialize the set $\mathbf{p}$ according to Lemma 6 and choose each $W_{i}^{*}$ except two be in form (82).

Now, let us choose the dual graph (this is petel graph. (see Figure $5 b$ where $n=3$ )) and consider

$$
\begin{gathered}
\mathbb{E}_{n, 0}\left\{\operatorname{det}\left(1-z Z_{1}^{\dagger} C_{-1} Z_{2}^{\dagger} C_{-2} \cdots Z_{n}^{\dagger} C_{-n}\right)^{-a} \prod_{i=1}^{n} \operatorname{det}\left(Z_{i} Z_{i}^{\dagger}\right)^{\alpha} e^{\sum_{m>0} \frac{1}{m} p_{m}^{(i)} \operatorname{tr}\left(\left(Z_{i} C_{i}\right)^{m}\right)}\right\}= \\
\sum_{\lambda} z^{|\lambda|}(a)_{\lambda} s_{\lambda}\left(C_{1} C_{-1} C_{2} C_{-2} \cdots C_{n} C_{-n}\right) \prod_{i=1}^{n} \frac{(N+\alpha)_{\lambda} s_{\lambda}\left(\mathbf{p}^{i}\right)}{(N)_{\lambda} s_{\lambda}\left(\mathbf{p}_{\infty}\right)}
\end{gathered}
$$

By Remark 16 we find all cases where the determinantal representations (20) or (21) exist.

Remark 17. Notice the following symmetry: the left-hand side produces the same right-hand side if we permute the set of exponents $\alpha_{1}, \ldots, \alpha_{n}, a-N$.

Example 19. Below $g=1,2, \ldots$ (For the case $g=1$ see Figure $1 a$ and zoomed Figure $3 a$; for $g=2$ see Figure 5e).

$$
\begin{gathered}
\mathbb{E}_{0,2 g}\left\{e^{\left.\sum_{m>0} \frac{1}{m} p_{m} \operatorname{tr}\left(U_{a_{1}} C_{a_{1}} U_{b_{1}} C_{b_{1}} U_{a_{1}}^{+} C_{-a_{1}} U_{b_{1}}^{+} C_{-b_{1}} \cdots U_{a_{g}} C_{a_{g}} U_{b_{g}} C_{b_{g}} U_{a_{g}}^{+} C_{-a_{g}} U_{b_{g}}^{+} C_{-b_{g}}\right)^{m}\right\}}\right. \\
=\sum_{\lambda}\left(\frac{\operatorname{dim} \lambda}{|\lambda| !}\right)^{-2 g} s_{\lambda}\left(W^{*}\right) s_{\lambda}(\mathbf{p})
\end{gathered}
$$

where

$$
W^{*}=C_{-a_{1}} C_{b_{1}} C_{a_{1}} C_{-b_{1}} \cdots C_{-a_{g}} C_{b_{g}} C_{a_{g}} C_{-b_{g}}
$$

In particular, if Spect $W^{*}=\mathbb{I}_{N, k}$, then

$$
\mathbb{E}_{0,2 g}\left\{\operatorname{det}\left(\mathbb{I}_{N}-z U_{a_{1}} C_{a_{1}} U_{b_{1}} C_{b_{1}} U_{a_{1}}^{\dagger} C_{-a_{1}} U_{b_{1}}^{\dagger} C_{-b_{1}} \cdots U_{a_{g}} C_{a_{g}} U_{b_{g}} C_{b_{g}} U_{a_{g}}^{\dagger} C_{-a_{g}} U_{b_{g}}^{\dagger} C_{-b_{g}}\right)^{-a}\right\}
$$




$$
=\sum_{\lambda} z^{|\lambda|}\left(\frac{\operatorname{dim} \lambda}{|\lambda| !}\right)^{2-2 g} \frac{(a)_{\lambda}(k)_{\lambda}}{\left((N)_{\lambda}\right)^{2 g}}
$$

Taking into account that

$$
\operatorname{dim} \lambda=\frac{\prod_{i<j}\left(h_{i}-h_{j}\right)}{\prod_{i=1}^{N} \Gamma\left(h_{i}+1\right)}
$$

we can interpret that (104) is a discrete beta-ensemble where $\beta=2-2 g$.

Exotic models. An example. There are some more tricky problems which can be solved which can be solved in steps. Let me consider the simplest example. Look at Lemma 3. Suppose $A$ and $B$ depend in any way on an additional matrix $Z_{1}$; in any case, however, their product has a familiar form:

$$
A=A\left(Z_{1}, Z_{1}^{\dagger}\right), \quad B=B\left(Z_{1}, Z_{1}^{\dagger}\right), \quad A B=Z_{1} C_{1} Z_{1}^{\dagger} C_{-1}
$$

Say, $A=Z_{1}^{a} e^{-Z_{1}^{\dagger}}, B=e^{Z_{1}^{\dagger}} Z_{1}^{\dagger} Z_{1}^{1-a}$ which looks horrible. However, applying sequentially the series ${ }_{0} F_{0}$, then (37), where $Z=Z_{1}$, and then (31), where $Z=Z_{2}$ one obtains

$$
\mathbb{E}_{2,0}\left\{e^{\operatorname{tr}\left(Z_{1} Z_{2}^{a} e^{-Z_{2}^{+}}\right)+\operatorname{tr}\left(Z_{1}^{\dagger} e^{Z_{2}^{+}} Z_{2}^{\dagger} Z_{2}^{1-a}\right)}\right\}=\sum_{d \geq 0} \sum_{\lambda \in Y_{d}} s_{\lambda}\left(\mathbb{I}_{N}\right) s_{\lambda}(C)=\operatorname{det}\left(\mathbb{I}_{N}-C\right)^{-1}
$$

It will be interesting to do the same with other ensembles of random matrices; Ginibre ensembles of real and quaternionic matrices; and ensembles of Hermitian matrices: complex, real and quaternionic.

\section{Discussion}

In this article, we examined matrix integrals of a certain type. We called them matrix models associated with children's drawings-the so-called dessin d'enfants. They include some well-known models that have found applications in the theory of information transfer and the theory of quantum chaos. We hope that our matrix integrals will be in demand. We think that these models are related to quantum integrable systems [38-40], but this topic is waiting for its development; we expect connections with [41-49].

Author Contributions: Conceptualization, A.O.; Investigation, N.A., A.O. and D.V.; Visualization, N.A. and D.V.; Writing-original draft, A.O.; Writing-review and editing, N.A., A.O. and D.V.

Funding: This research was funded by Russian Foundation for Basic Research: 18-01-00273a; Russian Foundation for Basic Research: 19-02-00815; Russian Foundation for Basic Research: 18-02-01081.

Acknowledgments: The authors are grateful to A. Gerasimov, M. Kazarian, S. Lando, Yu. Neretin, A. Morozov, A. Mironov, S. Natanzon and L. Chekhov for useful discussions. A.O. is grateful to A. Odzijewicz for his kind hospitality in Bialowezie and to E. Strahov, who turned his attention to independent Ginibre ensembles $[10,14,15]$. A.O. was partially supported by V.E. Zakharov's scientific school (Program for Support of Leading Scientific Schools), by RFBR grant 18-01-00273a. N.A. was partially supported by RFBR grant 19-02-00815. D.V. was partially supported by RFBR grant 18-02-01081.

Conflicts of Interest: The authors declare no conflict of interest.

\section{Appendix A. Partitions and Schur Functions}

Let us recall that the characters of the unitary group $\mathbb{U}(N)$ are labeled by partitionsand coincide with the so-called Schur functions [23]. A partition $\lambda=\left(\lambda_{1}, \ldots, \lambda_{n}\right)$ is a set of nonnegative integers $\lambda_{i}$ which are called parts of $\lambda$ and which are ordered as $\lambda_{i} \geq \lambda_{i+1}$. The number of non-vanishing parts of $\lambda$ is called the length of the partition $\lambda$, and will be denoted by $\ell(\lambda)$. The number $|\lambda|=\sum_{i} \lambda_{i}$ is called the weight of $\lambda$. The set of all partitions will be denoted by $\mathbb{P}$. 
The Schur function labelled by $\lambda$ may be defined as the following function in variables $x=\left(x_{1}, \ldots, x_{N}\right)$ :

$$
s_{\lambda}(x)=\frac{\operatorname{det}\left[x_{j}^{\lambda_{i}-i+N}\right]_{i, j}}{\operatorname{det}\left[x_{j}^{-i+N}\right]_{i, j}}
$$

in case $\ell(\lambda) \leq N$ and vanishes otherwise. One can see that $s_{\lambda}(x)$ is a symmetric homogeneous polynomial of degree $|\lambda|$ in the variables $x_{1}, \ldots, x_{N}$, and $\operatorname{deg} x_{i}=1, i=1, \ldots, N$.

Remark A1. In case the set $x$ is the set of eigenvalues of a matrix $X$, we also write $s_{\lambda}(X)$ instead of $s_{\lambda}(x)$.

There is a different definition of the Schur function as a quasi-homogeneous, non-symmetric polynomial of degree $|\lambda|$ in other variables, the so-called power sums, $\mathbf{p}=\left(p_{1}, p_{2}, \ldots\right)$, where $\operatorname{deg} p_{m}=m$.

For this purpose let us introduce

$$
s_{\{h\}}(\mathbf{p})=\operatorname{det}\left[s_{\left(h_{i}+j-N\right)}(\mathbf{p})\right]_{i, j},
$$

where $\{h\}$ is any set of $N$ integers, and where the Schur functions $s_{(i)}$ are defined by $e^{\sum_{m>0} \frac{1}{m} p_{m} z^{m}}=$ $\sum_{m \geq 0} s_{(i)}(\mathbf{p}) z^{i}$. If we put $h_{i}=\lambda_{i}-i+N$, where $N$ is not less than the length of the partition $\lambda$; then

$$
s_{\lambda}(\mathbf{p})=s_{\{h\}}(\mathbf{p}) .
$$

The Schur functions defined by (5) and by (A2) are equal, $s_{\lambda}(\mathbf{p})=s_{\lambda}(x)$, provided the variables $\mathbf{p}$ and $x$ are related by the power sums relation

$$
p_{m}=\sum_{i} x_{i}^{m}
$$

In case the argument of $s_{\lambda}$ is written as a non-capital fat letter the definition (A2), and we imply the definition (5) in case the argument is not fat and non-capital letter, and in case the argument is capital letter which denotes a matrix, then it implies the definition (5) with $x=\left(x_{1}, \ldots, x_{N}\right)$ being the eigenvalues.

It may be easily checked that

$$
s_{\lambda}(\mathbf{p})=(-1)^{|\lambda|} s_{\lambda^{\mathrm{tr}}}(-\mathbf{p})
$$

where $\lambda^{\text {tr }}$ is the partition conjugated to $\lambda$ (in [23] it is denoted by $\lambda^{*}$ ). The Young diagram of the conjugated partition is obtained by the transposition of the Young diagram of $\lambda$ with respect to its main diagonal. One gets $\lambda_{1}=\ell\left(\lambda^{\text {tr }}\right)$.

\section{Appendix B. Integrals over the Unitary Group}

Consider the following integral over the unitary group which depends on two semi-infinite sets of parameters $\mathbf{p}=\left(p_{1}, p_{2}, \ldots\right)$ and $\overline{\mathbf{p}}=\left(p_{1}^{*}, p_{2}^{*}, \ldots\right)$ :

$$
\begin{gathered}
I_{\mathbb{U}(N)}(\mathbf{p}, \overline{\mathbf{p}}):=\int_{\mathbb{U}(N)} e^{\operatorname{tr} V(\mathbf{p}, U)+\operatorname{tr} V\left(\mathbf{p}^{*}, U^{-1}\right)} d_{*} U= \\
\frac{1}{(2 \pi)^{N}} \int_{0 \leq \theta_{1} \leq \cdots \leq \theta_{N} \leq 2 \pi} \prod_{1 \leq j<k \leq N}\left|e^{i \theta_{j}}-e^{-i \theta_{k}}\right|^{2} \prod_{j=1}^{N} e^{\sum_{m>0} \frac{1}{m}\left(p_{m} e^{i m \theta_{j}}+p_{m}^{*} e^{-i m \theta_{j}}\right)} d \theta_{j} \\
V(\mathbf{p}, x):=\sum_{n>0} \frac{1}{n} p_{n} x^{n}
\end{gathered}
$$


Here $d_{*} U$ is the Haar measure of the group $\mathbb{U}(N)$ :

$$
d_{*} U=\frac{1}{(2 \pi)^{N}} \prod_{1 \leq j<k \leq N}\left|e^{i \theta_{j}}-e^{-i \theta_{k}}\right|^{2} \prod_{j=1}^{N} d \theta_{j}, \quad-\pi \leq \theta_{1}<\ldots \theta_{N} \leq \pi
$$

and $e^{i \theta_{1}}, \ldots, e^{i \theta_{N}}$ are the eigenvalues of $U \in \mathbb{U}(N)$. The exponential factors inside the integral may be treated as a perturbation of the Haar measure and parameters $\mathbf{p}, \mathbf{p}^{*}$ are called coupling constants by the analogy with quantum field theory problems.

Using the Cauchy-Littlewood identity

$$
\tau\left(\mathbf{p} \mid \mathbf{p}^{*}\right):=e^{\sum_{m=1}^{\infty} \frac{1}{m} p_{m}^{*} p_{m}}=\sum_{\lambda \in \mathbb{P}} s_{\lambda}\left(\mathbf{p}^{*}\right) s_{\lambda}(\mathbf{p})
$$

and the orthogonality of the irreducible characters of the unitary group

$$
\int s_{\lambda}(U) s_{\mu}\left(U^{-1}\right) d_{*} U=\delta_{\lambda, \mu}
$$

we obtain that

$$
I_{\mathbb{U}(n)}(\mathbf{p}, \overline{\mathbf{p}})=\sum_{\substack{\lambda \in \mathbb{P} \\ \ell(\lambda) \leq n}} s_{\lambda}(\mathbf{p}) s_{\lambda}(\overline{\mathbf{p}})
$$

which express the integral over unitary matrices as the "perturbation series in coupling constants".

The formula (A11) first appeared in [50] in the context of the study of Brezin-Gross-Witten model. It was shown there that the integral $I_{\mathbb{U}(n)}(\mathbf{p}, \overline{\mathbf{p}})$ may be related to the Toda lattice tau function of $[51,52]$ under certain restriction. Then, the series in the Schur functions (A11) may be related to the double Schur functions series found in [53-55].

\section{Appendix C. Geometrical Definition of Hurwitz Numbers}

In this presentation, we follow article [31].

The Hurwitz number is a characterisation of the branched covering of a surface with critical values of a prescribed topological type. Hurwitz numbers of oriented surfaces without boundaries were introduced by Hurwitz at the end of the 19th century. Later it turned out that they are closely related to the study of moduli spaces of Riemann surfaces [56], to integrable systems [57], to modern models of mathematical physics (matrix models) and to closed topological field theories [30]. In this paper we consider only Hurwitz numbers of compact surfaces without boundary.

Consider a branched covering $f: P \rightarrow \Sigma$ of degree $d$ of a compact surface without boundary. In the neighborhood of each point $z \in P$, the map $f$ is topologically equivalent to the complex map $u \mapsto u^{p}$, defined on a neighborhood $u \sim 0$ in $\mathbb{C}$. The number $p=p(z)$ is called the degree of the covering $f$ at the point $z$. The point $z \in P$ is said to be a branch point or critical point if $p(z) \neq 1$. There are only a finite number of critical points. The image $f(z)$ of a critical point $z$ is called the critical value of $f$ at $z$.

Let us associate with a point $s \in \Sigma$ all points $z_{1}, \ldots, z_{\ell} \in P$ for which $f\left(z_{i}\right)=s$. Let $p_{1}, \ldots, p_{\ell}$ be the degrees of the map $f$ at these points. Their sum $d=p_{1}+\cdots+p_{\ell}$ is equal to the degree $d$ of $f$. Thus, to each point $s \in S$ there corresponds a partition $d=p_{1}+\cdots+p_{\ell}$ of the number $d$. Having ordered the degrees $p_{1} \geq \cdots \geq p_{\ell}>0$ at each point $s \in \Sigma$, we introduce the Young diagram $\Delta^{s}=\left[p_{1}, \ldots, p_{\ell}\right]$ of weight $d$ with $\ell=\ell\left(\Delta^{s}\right)$ rows of length $p_{1} \ldots, p_{\ell}$ : $\Delta^{s}$ is called the topological type of the value $s$, and $s$ is a critical value of $f$ if and only if at least one of the row-lengths $p_{i}$ is greater than 1.)

Let us note that the Euler characteristics $\mathrm{E}(P)$ and $\mathrm{E}(\Sigma)$ of the surfaces $P$ and $\Sigma$ are related via the Riemann-Hurwitz relation:

$$
\mathrm{E}(P)=\mathrm{E}(\Sigma) d+\sum_{z \in P}(p(z)-1)
$$


or, equivalently,

$$
\mathrm{E}(P)=\mathrm{E}(\Sigma) d+\sum_{i=1}^{\mathrm{F}}\left(\ell\left(\Delta^{s_{i}}\right)-d\right)
$$

where $s_{1}, \ldots, s_{\mathrm{F}}$ are critical values.

We say that coverings $f_{1}: P_{1} \rightarrow \Sigma$ and $f_{2}: P_{2} \rightarrow \Sigma$ are equivalent if there exists a homeomorphism $F: P_{1} \rightarrow P_{2}$ such that $f_{1}=f_{2} F$; in case $P_{1}=P_{2}$ and $f_{1}=f_{2}$ the homeomorphism $F$ is called an automorphism of the covering. The set of all automorphisms of a covering $f$ form the group $\operatorname{Aut}(f)$ of finite order $|\operatorname{Aut}(f)|$. Equivalent coverings have isomorphic automorphism groups.

We present two illustrative examples.

Example 1. Let $\Sigma=\overline{\mathbb{C}}=\{z \in \mathbb{C}\} \cup \infty, P=P_{1}=P_{2}=\overline{\mathbb{C}}=\{u \in \mathbb{C}\} \cup \infty$ be Riemann spheres. Consider the branched covering $z(u)=f(u)=f_{1}(u)=f_{2}(u)=u^{3}$. This covering $f: P \rightarrow \Sigma$ has 2 critical values 0 and $\infty$ with Young diagrams from one row of length 3 . Automorphisms of the covering have the form $F(u)=u^{\sqrt[3]{1}}$. The group Aut $(f)$ is isomorphic to $\mathbb{Z} / 3 \mathbb{Z}$.

Example 2. Let $\Sigma=\overline{\mathbb{C}}=\{z \in \mathbb{C}\} \cup \infty$ and $P=P_{1}=P_{2}$-this is a pair of Riemann spheres; that is $P=P^{\prime} \cup P^{\prime \prime}$. where $P^{\prime}=\left\{u^{\prime} \in \mathbb{C}\right\} \cup \infty$ and $P^{\prime \prime}=\left\{u^{\prime \prime} \in \mathbb{C}\right\} \cup \infty$. Consider the branched covering $z\left(u^{\prime}\right)=f\left(u^{\prime}\right)=f_{1}\left(u^{\prime}\right)=f_{2}\left(u^{\prime}\right)=\left(u^{\prime}\right)^{3}, z\left(u^{\prime \prime}\right)=f\left(u^{\prime \prime}\right)=f_{1}\left(u^{\prime \prime}\right)=f_{2}\left(u^{\prime \prime}\right)=\left(u^{\prime \prime}\right)^{3}$. This covering $f: P \rightarrow \Sigma$ has two critical values 0 and $\infty$ with Young diagrams of two rows of length 3 . Automorphisms of the covering are generated by the following mappings:

1. $F\left(u^{\prime}\right)=\left(u^{\prime}\right) \sqrt[3]{1}, F\left(u^{\prime \prime}\right)=u^{\prime \prime}$.

2. $F\left(u^{\prime \prime}\right)=\left(u^{\prime \prime}\right)^{\sqrt[3]{1}}, F\left(u^{\prime}\right)=u^{\prime}$.

3. $F\left(u^{\prime}\right)=\left(u^{\prime \prime}\right), F\left(u^{\prime \prime}\right)=u^{\prime}$.

The group $\operatorname{Aut}\left(f_{i}\right)$ is isomorphic to $(\mathbb{Z} / 3 \mathbb{Z}) \otimes(\mathbb{Z} / 3 \mathbb{Z}) \otimes(\mathbb{Z} / 2 \mathbb{Z})$.

From now on, unless indicated otherwise, we will assume that the surface $\Sigma$ is connected. Let us choose points $s_{1}, \ldots, s_{\mathrm{F}} \in \Sigma$ and corresponding Young diagrams $\Delta^{1}, \ldots, \Delta^{\mathrm{F}}$ of weight $d$. Let $\Phi$ be the set of equivalence classes of the coverings for which $s_{1}, \ldots, s_{\mathrm{F}}$ is the set of all critical values, and $\Delta^{1}, \ldots, \Delta^{\mathrm{F}}$ are the topological types of these critical values. The Hurwitz number is the number

$$
H_{\Sigma}^{d}\left(\Delta^{1}, \ldots, \Delta^{\mathrm{F}}\right)=\sum_{f \in \Phi} \frac{1}{|\operatorname{Aut}(f)|} .
$$

It is easy to prove that the Hurwitz number is independent of the positions of the points $s_{1}, \ldots, s_{\mathrm{F}}$ on $\Sigma$. One can show that the right-hand side of (A13) depends only on the Young diagrams of $\Delta^{1}, \ldots, \Delta^{\mathrm{F}}$ and the Euler characteristic $\mathrm{E}=\mathrm{E}(\Sigma)$. Because of this sometimes we write $H_{\mathrm{E}(\Sigma)}^{d}\left(\Delta^{1}, \ldots, \Delta^{\mathrm{F}}\right)$ instead of $H_{\Sigma}^{d}\left(\Delta^{1}, \ldots, \Delta^{\mathrm{F}}\right)$.

If $\mathrm{F}=0$ we get an unbranched covering. We denote such Hurwitz number $H_{\mathrm{E}}\left(\left(1^{d}\right)\right)$.

Example 3. Let $f: \Sigma \rightarrow \mathbb{R P}^{2}$ be a covering without critical points. Then, if $\Sigma$ is connected, then $\Sigma=\mathbb{R P}^{2}, \operatorname{deg} f=1$ or $\Sigma=S^{2}, \operatorname{deg} f=2$. Therefore if $d=3$, then $\Sigma=\mathbb{R} \mathbb{P}^{2} \amalg \mathbb{R} \mathbb{P}^{2} \amalg \mathbb{R} \mathbb{P}^{2}$ or $\Sigma=\mathbb{R} \mathbb{P}^{2} \amalg S^{2}$. Thus $H_{1}\left(\left(1^{3}\right)\right)=\frac{1}{3 !}+\frac{1}{2 !}=\frac{2}{3}$.

\section{Appendix D. Combinatorial Definition of Hurwitz Numbers}

Consider the symmetric group (equivalently, the permutation group) $S_{d}$ and the equation

$$
\sigma_{1} \cdots \sigma_{\mathrm{F}} \rho_{1}^{2} \cdots \rho_{\mathrm{M}}^{2} \alpha_{1} \beta_{1} \alpha_{1}^{-1} \beta_{1}^{-1} \cdots \alpha_{\mathrm{H}} \beta_{\mathrm{H}} \alpha_{\mathrm{H}}^{-1} \beta_{\mathrm{H}}^{-1}=1,
$$

where $\sigma_{1}, \cdots, \sigma_{\mathrm{F}}, \rho_{1}, \cdots, \rho_{\mathrm{M}}, \alpha_{1}, \beta_{1}, \ldots, \alpha_{\mathrm{H}}, \beta_{\mathrm{H}} \in S_{d}$, and moreover $\sigma_{i} \in C_{\Delta^{i}}, i=1, \ldots, \mathrm{F}$, where $C_{\Delta^{i}}$ is the conjugacy class labeled by a partition $\Delta^{i}=\left(\Delta_{1}^{i}, \Delta_{2}^{i}, \ldots\right)$. The Hurwitz number is the number of solutions of Equation (A14) divided by $d$ ! (by the order of $S_{d}$ ). 
It can be proved that so introduced the (combinatorial) Hurwitz number coincides with the (geometric) Hurwitz number $H_{\mathrm{E}}\left(\Delta^{1}, \ldots, \Delta^{\mathrm{F}}\right)$ introduced in Appendix $\mathrm{C}$ where $\mathrm{E}=2-2 \mathrm{H}-\mathrm{M}$. (One can look at the base surface $\Sigma$ as a result of gluing $\mathrm{H}$ handles and M Möbius stripes to a sphere.

Consider the simplest example: $\mathrm{H}=0$ and $\mathrm{M}=1$; that is $\Sigma=\mathbb{R P}^{2}$ (real projective plane). Suppose $\mathrm{F}=0$; that is we deal with an unbranched covering. Suppose $d=3$; that is we consider 3-sheeted covering. Let us solve $\rho^{2}=1$, where $\rho \in S_{3}$. One gets 4 solutions: 3 transpositions of the set $1,2,3$ and one identity permutation. There are 3 ! permutations in $S_{3}$. As a result we get $H_{1}\left(\left(1^{3}\right)\right)=4 / 3 !=2 / 3$ as we got in the last example of the previous section.

In the same way one can consider Example 1 of the previous section. In this case $\mathrm{H}=\mathrm{M}=0$; that is $\mathrm{E}=2$; one gets the Riemann sphere with two branch points $(\mathrm{F}=2)$ and 3-sheeted covering with profiles $\Delta^{1}=\Delta^{2}=(3)$. We solve the equation $\sigma_{1} \sigma_{2}=1$, where both $\sigma_{1,2}$ consist of a single cycle of length 3 . There are two solutions $\sigma_{1}=\sigma_{2}^{-1}$ : one sends $1,2,3$ to $3,1,2$, the other sends $1,2,3$ to $2,3,1$. We get $H_{2}((3),(3))=2 / 3 !=1 / 3$.

Example 2 corresponds to $H_{2}((3,3),(3,3)), d=6$. One can complete the exercise and get an answer $H_{2}((3,3))=1 / z_{(3,3)}=1 / 18$, where $z_{\lambda}$ is given by (1). Actually, for any $d$ and for any pair of profiles one gets $H_{2}\left(\Delta^{1}, \Delta\right)=\delta_{\Delta^{1}, \Delta^{1}} 1 / z_{\Delta}$.

In [58,59] (and also in [60]) it was found that $H_{\mathrm{E}}\left(\Delta^{1}, \ldots, \Delta^{\mathrm{F}}\right)$ is given by formula (27).

\section{Appendix E. Differential Operators}

In [32] we develop (see also [32]) the work [61], which offers a beautiful generalization of the cut-and-join formula (MMN formula):

$$
\mathcal{W}^{\Delta}(\mathbf{p}) \cdot s_{\mu}(\mathbf{p})=\varphi_{\mu}(\Delta) s_{\mu}(\mathbf{p}),
$$

which describes the merging of pairs of branch points in the covering problem. Here $\mu=\left(\mu_{1}, \mu_{2}, \ldots\right)$ and $\Delta=\left(\Delta_{1}, \ldots, \Delta_{\ell}\right)$ are Young diagrams $(\Delta$ is the ramification profile of one of branch points; for simplicity, we consider the case where $|\mu|=|\Delta|)$, $s_{\mu}$ is the Schur function. Differential operators $\mathcal{W}^{\Delta}(\mathbf{p})$ generalize the operators of "additional symmetries" [62] in the theory of solitons and commute with each other for different $\Delta$. In the work ([63]), it was noted that if they are written in the so-called Miwa variables, that is, in terms of the eigenvalues of the matrix $X$ such that $p_{m}=\operatorname{tr}\left(X^{m}\right)$; then the generalized cut-and-join formula is written very compactly and beautifully:

$$
\mathcal{W}^{\Delta} \cdot s_{\mu}(X)=\varphi_{\mu}(\Delta) s_{\mu}(X)
$$

where

$$
\mathcal{W}^{\Delta}=\frac{1}{z_{\Delta}} \operatorname{tr}\left(D^{\Delta_{1}}\right) \cdots \operatorname{tr}\left(D^{\Delta_{\ell}}\right),
$$

and the factor $z_{\Delta}$ is given by (1), $D$ is

$$
D_{a, b}=\sum_{c=1}^{N} X_{a, c} \frac{\partial}{\partial X_{b, c}}
$$

As G.I. Olshansky pointed out to us, this type of formula appeared in the works of Perelomov and Popov [64-66] and describe the actions of the Casimir operators in the representaion $\lambda$; see also [67], Section 9.

We propose a generalization of this relation, which in our case is constructed using a child's drawing of a constellation (dessin d'enfants, or a map in terminology [28]. In fact, we are considering a modification in which the vertices are replaced by small disks-"stars"). This topic will be be studied in more detail in the next article. Here we restrict ourselves only to a reference to important beautiful works [41-45]. 
(i) One can interpret the Gaussian integral as the integral of $n$-component two-dimensional charged bosonic fields $Z_{i}$ and $Z_{i}^{+}$:

$$
\int\left(Z_{i}^{\dagger}\right)_{a, b}\left(Z_{j}\right)_{b^{\prime}, a^{\prime}} d \Omega=<\left(Z_{i}^{\dagger}\right)_{a, b}\left(Z_{j}\right)_{b^{\prime}, a^{\prime}}>=\frac{1}{N} \delta_{a, a^{\prime}} \delta_{b, b^{\prime}} \delta_{i, j}
$$

for $i, j=1, \ldots, n, \quad a, b=1, \ldots, N$.

The Fock space of these fields is all possible polynomials from the matrix elements of the matrices $Z_{1}, \ldots, Z_{n}$.

The operators $\left(Z_{i}\right)_{a b}$ can be considered creation operators, and the operators

$$
\left(Z_{i}^{\dagger}\right) \rightarrow \frac{1}{N} \partial_{i}, \quad\left(\partial_{i}\right)_{a, b}=\frac{1}{N} \frac{\partial}{\partial Z_{b, a}}
$$

ellimination operators that act in this space. The integrands in our integrals should be considered anti-ordered, that is, all ellimination operators (all derivatives) considered to be moved to the left, while the matrix structure is considered to be preserved. We will denote this is anti-ordering of some $A$ by the symbol :: $A::$, where $A$ is a polynomial of matrix elements of the matrices

From this point of view, on different sides of the ribbon of $\tilde{\Gamma}$ with the number $i$ we place the canonically conjugated coordinates $Z_{i}$ and momenta $\partial_{Z_{i}}$.

We recall that all partions throught the paper have the same weight $d$.

(ii) Then, for example, the relation we get

$$
:: \tau_{g}\left(M_{1}, \ldots, M_{\mathrm{F}}\right)::=\sum_{d=0}^{\infty} \sum_{\substack{\Delta^{1}, \ldots, \Delta^{\mathrm{V}} \\\left|\Delta^{1}\right|=\cdots=\left|\Delta^{\mathrm{V}}\right|=d}} H_{\Sigma^{\prime}}\left(g \mid \Delta^{1}, \ldots, \Delta^{\mathrm{V}}\right) C\left(\Delta^{1}, \ldots, \Delta^{\mathrm{V}}\right) \text {, }
$$

We give another relation:

$$
\left(:: \prod_{i=1}^{\mathrm{F}} s_{\lambda^{i}}\left(M_{i}\right)::\right) \cdot 1=\delta_{\lambda^{1}, \ldots, \lambda^{\mathrm{F}}}\left(\frac{\operatorname{dim} \lambda}{d !}\right)^{-n} \prod_{i=1}^{\mathrm{V}} s_{\lambda}\left(W_{i}^{*}\right)
$$

where $\lambda^{1}, \ldots, \lambda^{\mathrm{F}}=\lambda$ is a set of Young diagrams, and where $\delta_{\lambda^{1}, \ldots, \lambda^{\mathrm{F}}}$ is equal 1, if $\lambda^{1}=\cdots=\lambda^{\mathrm{F}}=\lambda$ and is equal to 0 otherwise.

It looks like a simple rewrite, but can be helpfully used. Let us derive a beautiful formula (Theorem 5.1 in [63]), namely (A16) (and see also articles [41-46]).

In order to do this we should use the freedom to choose the source matrices:

(iii) For a partition $\Delta=\left(\Delta_{1}, \ldots, \Delta_{\ell}\right)$ and a face monodromy $M_{i}$ and a star monodromy $W_{i}^{*}$, let us introduce notations

$$
\begin{aligned}
& \mathcal{M}_{i}^{\Delta^{i}}=\operatorname{tr}\left(\left(M_{i}\right)^{\Delta_{1}^{i}}\right) \cdots \operatorname{tr}\left(\left(M_{i}\right)^{\Delta_{\ell}^{i}}\right) \\
& \mathcal{C}_{i}^{\Delta^{i}}=\operatorname{tr}\left(\left(W_{i}^{*}\right)^{\Delta_{1}^{i}}\right) \cdots \operatorname{tr}\left(\left(W_{i}^{*}\right)^{\Delta_{\ell}^{i}}\right)
\end{aligned}
$$

We can write

$$
\begin{gathered}
N^{n d} \int\left[\prod_{i=1}^{\mathrm{F}} \frac{\mathcal{M}_{i}^{\tilde{\Delta}^{i}}}{z_{\tilde{\Delta}^{i}}}\right] d \Omega \\
=\sum_{\Delta^{1}, \ldots, \Delta^{\mathrm{v}}} H_{\Sigma}\left(\tilde{\Delta}^{1}, \ldots, \tilde{\Delta}^{\mathrm{F}}, \Delta^{1}, \ldots, \Delta^{\mathrm{V}}\right)\left[\prod_{i=1}^{\mathrm{V}} \mathcal{C}_{i}^{\Delta^{i}}\right]
\end{gathered}
$$

Let us write the most general generating function for Hurwitz numbers which was obtained in [31]:

$$
N^{n d} \int\left[\prod_{i=1}^{\mathrm{F}_{1}} \frac{\mathcal{M}_{i}^{\tilde{\Delta}^{i}}}{z_{\tilde{\Delta}^{i}}}\right]\left(\prod_{i=\mathrm{F}_{1}+1}^{\mathrm{F}_{2}} \mathfrak{M}\left(M_{i}\right)\right)\left(\prod_{i=\mathrm{F}_{2}+2, \mathrm{~F}_{2}+4, \ldots}^{\mathrm{F}} \mathfrak{H}\left(M_{i-1}, M_{i}\right)\right) d \Omega
$$




$$
=\sum_{\substack{\Delta^{1}, \ldots, \Delta^{\mathrm{V}} \\ \Delta^{1}|=\cdots=| \Delta^{\mathrm{V}} \mid=d}} H_{\tilde{\Sigma}}\left(\tilde{\Delta}^{1}, \ldots, \tilde{\Delta}^{\mathrm{F}_{1}}, \Delta^{1}, \ldots, \Delta^{\mathrm{V}}\right) C\left(\Delta^{1}, \ldots, \Delta^{\mathrm{V}}\right),
$$

where

$$
H_{\tilde{\Sigma}}\left(\tilde{\Delta}^{1}, \ldots, \tilde{\Delta}^{\mathrm{F}_{1}}, \Delta^{1}, \ldots, \Delta^{\mathrm{V}}\right)=\sum_{\lambda \in \mathcal{P}}\left(\frac{\operatorname{dim} \mu}{d !}\right)^{\mathrm{F}-n+V-2 h-m} \varphi_{\mu}\left(\tilde{\Delta}^{1}\right) \cdots \varphi_{\mu}\left(\tilde{\Delta}^{\mathrm{F}_{1}}\right) \varphi_{\mu}\left(\Delta^{1}\right) \cdots \varphi_{\mu}\left(\Delta^{\mathrm{V}}\right)
$$

where $h=\frac{1}{2}\left(\mathrm{~F}-\mathrm{F}_{2}\right)$ is the number of handles and $m=\mathrm{F}_{2}-\mathrm{F}_{1}$ is the number of Moebius stripes glued to $\Sigma$ where the graph $\tilde{\Gamma}$ (modified dessin d'enfants) was drawn. The Euler characteristic of $\tilde{\tilde{\Sigma}}$ is $\mathrm{F}-n+V-2 h-m=\mathrm{F}_{1}-n+\mathrm{V}$. Hurwitz number (A28) counts the coverings of $\tilde{\tilde{\Sigma}}$ with branching profiles $\tilde{\Delta}^{1}, \ldots, \tilde{\Delta}^{\mathrm{F}_{1}}, \Delta^{1}, \ldots, \Delta^{\mathrm{V}}$.

Let us multiply the both sides of (A26) by

$$
\prod_{i=k+1}^{\mathrm{F}_{1}} \frac{\operatorname{dim} \mu^{i}}{d !} \varphi_{\mu^{i}}\left(\tilde{\Delta}^{i}\right) z_{\tilde{\Delta}^{i}}
$$

(where $k \leq \mathrm{F}$ ), and then sum the both sides (A26) and (A27) over $\tilde{\Delta}^{i}, i=k+1, \ldots, \mathrm{F}_{1}$, taking into account

$$
s_{\mu}(X)=\frac{\operatorname{dim} \mu}{d !} \sum_{\Delta} \varphi_{\mu}(\Delta) \mathcal{X}^{\Delta},
$$

when evaluating (A26), where

$$
\mathcal{X}^{\Delta}=\operatorname{tr}\left((X)^{\Delta_{1}}\right) \cdots \operatorname{tr}\left((X)^{\Delta_{\ell}}\right)
$$

and the orthogonality relation (11) when evaluating (A27). We obtain

$$
\begin{aligned}
\int N^{n d}\left[\prod_{i=1}^{k} \frac{\mathcal{M}_{i}^{\tilde{i}^{i}}}{z_{\tilde{\Delta}^{i}}}\right]\left(\prod_{i=k+\mathrm{M}+1}^{k+\mathrm{M}+m} \mathfrak{M}\left(M_{i}\right)\right)\left(\prod_{i=k+\mathrm{M}+m+2, k+\mathrm{M}+m+4, \ldots .}^{\mathrm{F}} \mathfrak{H}\left(M_{i-1}, M_{i}\right)\right) \prod_{i=k+1}^{k+\mathrm{M}} s_{\mu^{i}}\left(M_{i}\right) d \Omega \\
=\delta_{\mu^{k+1}, \ldots, \mu^{\mathrm{F}-k}}\left(\frac{\operatorname{dim} \mu}{d !}\right)^{k-n-2 h-m} \varphi_{\mu}\left(\tilde{\Delta}^{1}\right) \cdots \varphi_{\mu}\left(\tilde{\Delta}^{k}\right)\left[\prod_{i=1}^{\mathrm{v}} s_{\lambda}\left(W_{i}^{*}\right)\right]
\end{aligned}
$$

where $2 h=\mathrm{F}-\mathrm{F}_{1}-m \mathrm{M}$

Remark A2. Suppose that the edges of the graph $\Gamma$ can be painted like a chessboard in black and white faces so that the face of one color borders only the faces of a different color. Then the matrices from the set $\left\{Z^{\dagger}\right\}$ (i.e., differential operators) can be assigned to the sides of the edges of white faces, that is, the matrices from the set $\{Z\}$ to the sides of black faces. In this case, the monodromies of the white faces will be those differential operators which will act on the monodromy of black faces.

The most natural and simple case is the following "polarization": Suppose that $M_{i}, i=k+1, \ldots, \mathrm{F}_{1}$ are black faces and the rest part of the face monodromies $M_{i}$ are while faces (see Remark A2).

(I) Let $m=h=0$. Take as a graph $\tilde{\Gamma}$ a child's drawing - sunflower with $n$ white petals drawn on the background of black night sky. See (b) in the Figure 5 for $\Gamma$ with 3 petals as an example. There is 1 vertex of $\Gamma$ which inflated and we get a small disk as the center of sunflower. We have $n+1$ faces of $\Gamma$ : $n$ petals, and the big and a big face, embracing all the petals and containing infinity. Then we place all "momentums" inside the petals:

$$
M_{i}=C_{-i} Z_{i}^{\dagger}, i=1, \ldots, n
$$


Then all "coordinates" (the collections of $\left\{\left(Z_{i}\right)_{a}, b\right\}$ ) are placed on the other side of the ribbons, they are places along the boundary of the big embracing black face:

$$
M_{n+1}=Z_{1} C_{1} \cdots Z_{n} C_{n}
$$

See Figure $5 b$ as an example.

Let remove the sign tilde above Young diagrams, then,

$$
\int N^{n d}\left[\prod_{i=1}^{n} \frac{\mathcal{M}_{i}^{\Delta^{i}}}{z_{\Delta^{i}}}\right] s_{\mu}\left(Z_{1} C_{1} \cdots Z_{n} C_{n}\right) d \Omega=\varphi_{\mu}\left(\Delta^{1}\right) \cdots \varphi_{\mu}\left(\Delta^{n}\right) s_{\mu}\left(C_{-1} C_{1} \cdots C_{-n} C_{n}\right)
$$

It is equivalent to

$$
\mathcal{W}_{C_{-1}}^{\Delta^{1}} \cdots \mathcal{W}_{C_{-n}}^{\Delta^{n}} \cdot s_{\mu}\left(Z_{1} C_{1} \cdots Z_{n} C_{n}\right)=\varphi_{\mu}\left(\Delta^{1}\right) \cdots \varphi_{\mu}\left(\Delta^{n}\right) s_{\mu}\left(C_{-1}\left(Z_{1}\right) C_{1} \cdots C_{-n}\left(Z_{n}\right) C_{n}\right),
$$

where each $N \times N$ matrix $C_{-i}$ can now depend, for example, polynomially on $Z_{i}, i=1, \ldots, n$ and where

$$
\mathcal{W}_{C_{-i}}^{\Delta^{i}}=: \operatorname{tr}\left(\left(C_{-i}\left(Z_{i}\right) \partial_{i}\right)^{\Delta_{1}^{i}}\right) \cdots \operatorname{tr}\left(\left(C_{-i}\left(Z_{i}\right) \partial_{i}\right)^{\Delta_{\ell}^{i}}\right):,
$$

where each $C_{-i} \partial_{i}$ is a matrix whose entries are differential operators, more precisely, are the following vector fields:

$$
\left(C_{-i} \partial_{i}\right)_{a, b}:=\sum_{c=1}^{N}\left(C_{-i}\right)_{a, c} \frac{\partial}{\partial\left(Z_{i}\right)_{b, c}}
$$

The normal ordering indicated by two dots is the same here as in [63] - that is, while maintaining the matrix structure, the derivative operators do not act on $C_{-i}=C_{-i}\left(Z_{i}\right)$. Note that the normal ordering procedure is necessary in order the Equation (A34) was equivalent to the equality (A33)!

The ordering is the same as in [63]: keeping the matrix structure the derivatives do not act on $C_{-i}=C_{-i}\left(Z_{i}\right)$. Notice that the ordering is necessary to relate (A34) to (A33)!

If we now take the case $n=1$ (one petal), and in addition, $C_{-i}=Z_{i}$, then we get the desired formula (A16).

Take another example with the same graph $\tilde{\Gamma}$ with the same monodromies. However, let $k=0$. In this case the integral (A33) can be re-written as the relation

$$
\hat{\mathfrak{M}}_{1} \cdots \hat{\mathfrak{M}}_{m} \hat{\mathfrak{H}}_{1} \cdots \hat{\mathfrak{H}}_{h} \cdot s_{\mu}\left(Z_{1} C_{1} \cdots Z_{n} C_{n}\right)=\left(\frac{\operatorname{dim} \mu}{|\mu| !}\right)^{-2 h-m-n} s_{\mu}\left(C_{-1}\left(Z_{1}\right) C_{1} \cdots C_{-n}\left(Z_{n}\right) C_{n}\right),
$$

where

$$
\begin{aligned}
& \hat{\mathfrak{M}}_{i}=: e^{\frac{1}{2} \sum_{j>0} \frac{1}{j}\left(\operatorname{tr}\left(C_{-i} \partial_{i}\right)^{j}\right)^{2}+\sum_{j>0, \text { odd }} \frac{1}{j} \operatorname{tr}\left(\left(C_{-i} \partial_{i}\right)^{j}\right)}:, \quad i=1, \ldots, m, \\
& \hat{\mathfrak{H}}_{i}=: e^{\sum_{j>0} \frac{1}{j} \operatorname{tr}\left(\left(C_{1-m-2 i} \partial_{m+2 i-1}\right)^{j}\right) \operatorname{tr}\left(\left(C_{-m-2 i} \partial_{m+2 i}\right)^{j}\right)}:, \quad i=1, \ldots, h .
\end{aligned}
$$

Remark A3. When $C_{-i}=Z_{i}, i=1, \ldots, n$ both equalities (A34) and (A37) describe eigenvalue problems for the corresponding Hamiltonians in the two-dimensional bosonic theory. Perhaps a comparison with the case analyzed by Dubrovin is appropriate. This is the case $n=1, \mathrm{~F}=1, \mathcal{W}^{(n)}$. In this case, the operators $\mathcal{W}^{(n)}, n=1,2, \ldots$ are the dispersionless Hamiltonians KdV equations [68].

Remark A4. The case $C_{-i}$ does not depend is also interesting in case the monodromies of the stars are degenerate matrices, then the whole intergal is related to the integration over rectanguler matrices. As an example one can choose $C_{-1} C_{1} \ldots C_{-n} C_{n}$ in (A33) as diag $(1,1, \ldots, 1,0,0, \ldots, 0)$. Then we get the Pochhhamer symbol in the right-hand side which allows one to related the whole integral to the hypergeometric tau function [24]. It will be discussed in a more detailed text where we plan to relate out topic to certain topics in [41-46]. 
Another example. $\Gamma$ has 2 vertices which are connected by 4 edges; see Figure $4 \mathrm{~d}$. We

$$
\begin{aligned}
& : \mathbf{p}_{\Delta^{1}}\left(\partial_{1} C_{-1} \partial_{2} C_{-2}\right) \mathbf{p}_{\Delta^{2}}\left(\partial_{3} C_{-3} \partial_{4} C_{-4}\right):\left(s_{\lambda}\left(Z_{1} C_{1} Z_{4} C_{4}\right) s_{\mu}\left(Z_{2} C_{2} Z_{3} C_{3}\right)\right) \\
& =\delta_{\lambda, \mu}\left(\frac{\operatorname{dim} \mu}{d !}\right)^{-2} \varphi_{\mu}\left(\Delta^{1}\right) \varphi_{\mu}\left(\Delta^{2}\right) s_{\mu}\left(C_{1} C_{-4} C_{3} C_{-2}\right) s_{\mu}\left(C_{4} C_{-1} C_{2} C_{-3}\right)
\end{aligned}
$$

In particular, if one takes $C_{3}=C_{4}=C$ and $C_{-1}=C_{-1}\left(Z_{2}\right), C_{-2}=C_{-2}\left(Z_{1}\right)$, $C_{-3}=C_{-3}\left(Z_{3}\right), C_{-4}=C_{-4}\left(Z_{4}\right)$ he gets

$$
\begin{aligned}
& : \mathbf{p}_{\Delta^{1}}\left(C_{-2}\left(Z_{1}\right) \partial_{1} C_{-1}\left(Z_{2}\right) \partial_{2}\right) \mathbf{p}_{\Delta^{2}}\left(\partial_{3} C_{-3}\left(Z_{3}\right) \partial_{4} C_{-4}\left(Z_{4}\right)\right): s_{\lambda}\left(Z_{1} C_{1} Z_{4} C\right) s_{\mu}\left(Z_{2} C_{2} Z_{3} C\right) \\
& =\delta_{\lambda, \mu}\left(\frac{\operatorname{dim} \mu}{d !}\right)^{-2} \varphi_{\mu}\left(\Delta^{1}\right) \varphi_{\mu}\left(\Delta^{2}\right) s_{\mu}\left(C_{-2}\left(Z_{1}\right) C_{1} C_{-4}\left(Z_{4}\right) C\right) s_{\mu}\left(C_{-1}\left(Z_{2}\right) C_{2} C_{-3}\left(Z_{3}\right) C\right)
\end{aligned}
$$

In particular, if one takes $C_{3}=C_{4}=C$ and $C_{-1}=Z_{2}, C_{-2}=Z_{1}, C_{-3}=Z_{3}, C_{-4}=Z_{4}$ (Euler fields) he gets an eigenvalue problem:

$$
\begin{aligned}
& : \mathbf{p}_{\Delta^{1}}\left(Z_{1} \partial_{1} Z_{2} \partial_{2}\right) \mathbf{p}_{\Delta^{2}}\left(\partial_{3} Z_{3} \partial_{4} Z_{4}\right):\left(s_{\lambda}\left(Z_{1} C_{1} Z_{4} C\right) s_{\mu}\left(Z_{2} C_{2} Z_{3} C\right)\right) \\
& =\delta_{\lambda, \mu}\left(\frac{\operatorname{dim} \mu}{d !}\right)^{-2} \varphi_{\mu}\left(\Delta^{1}\right) \varphi_{\mu}\left(\Delta^{2}\right) s_{\mu}\left(Z_{1} C_{1} Z_{4} C\right) s_{\mu}\left(Z_{2} C_{2} Z_{3} C\right)
\end{aligned}
$$

In case $C_{-1}=C_{-2}=C_{-3}=C_{-4}=\mathbb{I}_{N}$ we get

$$
\begin{gathered}
: \mathbf{p}_{\Delta^{1}}\left(\partial_{1} \partial_{2}\right) \mathbf{p}_{\Delta^{2}}\left(\partial_{3} \partial_{4}\right):\left(s_{\lambda}\left(Z_{1} C_{1} Z_{4} C_{4}\right) s_{\mu}\left(Z_{2} C_{2} Z_{3} C_{3}\right)\right) \\
=\delta_{\lambda, \mu} \varphi_{\mu}\left(\Delta^{1}\right) \varphi_{\mu}\left(\Delta^{2}\right) s_{\mu}\left(C_{1} C_{3}\right) s_{\mu}\left(C_{2} C_{4}\right)
\end{gathered}
$$

Now we consider another example $n=4$ with the graph obtained from the graph (a) in the Figures 1 and 3 drawn on the torus by doubling the edges: instead of each edge we draw two ones. We have $\Gamma$ with one vertex, four edges and three faces and obtain

$$
\begin{gathered}
: \mathbf{p}_{\Delta^{1}}\left(\partial_{1} C_{-1} \partial_{3} C_{-3}\right) \mathbf{p}_{\Delta^{1}}\left(\partial_{2} C_{-2} \partial_{4} C_{-4}\right): s_{\lambda}\left(Z_{1} C_{1} Z_{2} C_{2} Z_{3} C_{3} Z_{4} C_{4}\right) \\
=s_{\lambda}\left(C_{1} C_{-2} C_{4} C_{-1} C_{3} C_{-4} C_{2} C_{-3}\right)
\end{gathered}
$$

Take $C_{-1}=C_{-1}\left(Z_{3}\right), C_{-2}=C_{-2}\left(Z_{2}\right), C_{-3}=C_{-3}\left(Z_{1}\right), C_{-4}=C_{-4}\left(Z_{4}\right)$ and $C_{2}=C_{4}=C$. As an example we obtain

$$
\begin{gathered}
: \mathbf{p}_{\Delta^{1}}\left(Z_{1} \partial_{1} Z_{3} \partial_{3}\right) \mathbf{p}_{\Delta^{1}}\left(\partial_{2} Z_{2} \partial_{4} Z_{4}\right): s_{\lambda}\left(Z_{1} C_{1} Z_{2} C Z_{3} C_{3} Z_{4} C\right) \\
=\left(\frac{\operatorname{dim} \lambda}{d !}\right)^{-2} \varphi_{\lambda}\left(\Delta^{1}\right) \varphi_{\lambda}\left(\Delta^{2}\right) s_{\lambda}\left(Z_{1} C_{1} Z_{2} C Z_{3} C_{3} Z_{4} C\right)
\end{gathered}
$$

(iv) Let us notice that if we take a dual graph to the sunflower graph with $n=1$ (dual to one petal $\Gamma$, which is just a line segment; see Figure $2 a, b)$, in this case we have one face and two vertices, we get a version of the Capelli-type relation. Then it is a task to compare explicitly such relations with beautiful results [43-46].

(v) There are several allusions to the existence of interesting structures related to quantum integrability. First, as noted in [31] by this appearance 2D Yang-Mills theory [40]. See also possible connection to [48]. Then the appearance of the Yangians in works [41,42] which, we hope, can be related to our subject. And finally, the work [68].

(vi) There is a direct similarity between integrals over complex matrices and integrals over unitary matrices. However, from our point of view direct anologues of the relations in the present 
paper are more involved in the case of unitary matrices. In particular, Hurwitz numbers are replaced by a special combination of these numbers.

\section{References}

1. Vilenkin, N.Y.; Klimyk, A.U. Representation of Lie Groups and Special Functions; Volume 3: Classical and Quantum Groups and Special Functions; Kluwer Academic Publishers: Dordrecht, The Netherlands, 1992.

2. $\quad \mathrm{t}^{\prime}$ Hooft, G. A planar diagram theory for strong interactions. Nucl. Phys. 1974, 72, 461-473.

3. Itzykson, C.; Zuber, J.-B. The planar approximation. II. J. Math. Phys. 1980, 21, 411.

4. Kazakov, V.A.; Kostov, I.K.; Nekrasov, N. D-particles, Matrix Integrals and KP hierachy. Nucl. Phys. 1999, 557, 413-442. [CrossRef]

5. Brezin, E.; Kazakov, V.A. Exactly solvable field theories of closed strings. Phys. Lett. 1990, B236, 144-150. [CrossRef]

6. Gross, D.J.; Migdal, A.A. A nonperturbative treatment of two-dimensional quantum gravity. Nucl. Phys. B 1990, 340, 333-365. [CrossRef]

7. Collins, B.; Nechita, I. Random matrix techniques in quantum information theory. J. Math. Phys. 2016, 57, 015215. [CrossRef]

8. Preskill, J. Lecture Notes. Available online: http://www.theory.caltech.edu/ preskill/ph219/index.html\# lecture (accessed on 3 August 2020).

9. Tulino, A.M.; Verdu, S. Random Matrix Theory and Wireless Communications. Foundations and Trends in Communications and Information Theory; Now Publishers Inc.: Breda, The Netherlands, 2004; Volume 1.

10. Akemann, G.; Ipsen, J.R.; Kieburg, M. Products of Rectangular Random Matrices: Singular Values and Progressive Scattering. Phys. Rev. E 2013, 88, 052118. [CrossRef]

11. Akemann, G.; Checinski, T.; Kieburg, M. Spectral correlation functions of the sum of two independent complex Wishart matrices with unequal covariances. J. Phys. A Math. Theor. 2016, 49, 315201. [CrossRef]

12. Akemann, G.; Strahov, E. Hard edge limit of the product of two strongly coupled random matrices. Nonlinearity 2016, 29, 3743. [CrossRef]

13. Ipsen, J.R. Products of Independent Gaussian Random Matrices. arXiv 2015, arXiv:1510.06128;

14. Strahov, E. Dynamical correlation functions for products of random matrices. arXiv 2015, arXiv:1505.02511.

15. Strahov, E. Differential equations for singular values of products of Ginibre random matrices. arXiv 2014, arXiv:1403.6368.

16. Ambjørn, J.; Chekhov, L. The matrix model for dessins d'enfants. arXiv 2014, arXiv:1404.4240.

17. Kazakov, V.A.; Staudacher, M.; Wynter, T. Character Expansion Methods for Matrix Models of Dually Weighted Graphs. Commun. Math. Phys. 1996, 177, 451-468. [CrossRef]

18. Kazakov, V.A.; Staudacher, M.; Wynter, T. Almost Flat Planar Diagrams. Commun. Math. Phys. 1996, $179,235-256$

19. Kazakov, V.A.; Staudacher, M.; Wynter, T. Exact Solution of Discrete Two-Dimensional $R^{2}$ Gravity. Nucl. Phys. 1996, B471, 309-333

20. Kazakov, V.A. Solvable Matrix Models. arXiv 2000, arXiv:hep-th/0003064.

21. Alexandrov, A. Matrix models for random partitions. Nucl. Phys. B 2011, 851, 620-650. [CrossRef]

22. Ambjørn, J.; Chekhov, L.O. The matrix model for hypergeometric Hurwitz number. Theor. Math. Phys. 2014, 81, 1486-1498.

23. Macdonald, I.G. Symmetric Functions and Hall Polynomials, 2nd ed.; Clarendon Press: Oxford, UK; New York, NY, USA, 1995.

24. Orlov, A.Y.; Scherbin, D.M. Fermionic representation for basic hypergeometric functions related to Schur polynomials. arXiv 2000, arXiv:nlin/0001001.

25. Orlov, A.Y.; Scherbin, D.M. Hypergeometric solutions of soliton equations. Theor. Math. Phys. 2001, 128, 906-926. [CrossRef]

26. Orlov, A.Y. New solvable matrix integrals. Intern. J. Mod. Phys. 2004, 19 (Suppl. S2), 276-293. [CrossRef]

27. Orlov, A.Y.; Shiota, T.; Takasaki, K. Pfaffian structures and certain solutions to BKP hierarchies I. Sums over partitions. arXiv 2012, arXiv:math-ph/12014518.

28. Lando, S.K.; Zvonkin, A.K. Graphs on Surfaces and Their Applications; Encyclopaedia of Mathematical Sciences; Zagier, D., Ed.; Springer: New York, NY, USA, 2004; Volume 141. 
29. Okounkov, A.; Pandharipande, R. Gromov-Witten theory, Hurwitz theory and completed cycles. Ann. Math. 2006, 163, 517. [CrossRef]

30. Dijkgraaf, R. Mirror Symmetry and Elliptic Curves, The Moduli Space of Curves; Dijkgraaf, R., Faber, C., van der Geer, G., Eds.; Progress in Mathematics; Birkhauser: Basel, Switzerland, 1995; Volume 129.

31. Natanzon, S.M.; Orlov, A.Y. Hurwitz numbers from matrix integrals over Gaussian measure. arXiv 2020, arXiv:2002.00466.

32. Natanzon, S.M.; Orlov, A.Y. Hurwitz numbers from Feynman diagrams. Theor. Math. Phys. 2020, 204, 1172-1199.

33. Chekhov, L.O. The Harer-Zagier recursion for an irregular spectral curve. J. Geom. Phys. 2016, 110, $30-43$. [CrossRef]

34. Orlov, A.Y. Hurwitz numbers and products of random matrices. Theor. Math. Phys. 2017, 192, $1282-1323$. [CrossRef]

35. Orlov, A.Y. Links between quantum chaos and counting problems. In Geometric Methods in Physics XXXVI, Trends in Mathematics; Kielanowski, P., Odzijewicz, A., Previato, E., Eds.; Birkhauser: Cham, Switzerland, 2019; pp. 355-373.

36. Adrianov, N.M.; Amburg, N.Y.; Dremov, V.A.; Kochetkov, Y.Y.; Kreines, E.M.; Levitskaya, Y.A.; Nasretdinova, V.F.; Shabat, G.B. Catalog of dessins d'enfants with $\leq 4$ edges. J. Math. Sci. 2009, 158, 22-80. [CrossRef]

37. Natanzon, S.M.; Orlov, A.Y. Integals of tau functions. arXiv 2019, arXiv:1911.02003.

38. Migdal, A.A. Recursion equations in gauge field theories. JETP 1975, 42, 413.

39. Rusakov, B.Y. Loop avareges and partition functions in $U(N)$ gauge theory on two-dimensional manifold. Mod. Phys. Lett. A 1990, 5, 693-703. [CrossRef]

40. Witten, E. On Quantum Gauge Theories in Two Dimensions. Com. Math. Phys. 1991, 141, 153-209. [CrossRef]

41. Olshanski, G.I. Yangians and universal enveloping algebras. J. Soviet Math. 1989, 47, 2466-2473. [CrossRef]

42. Olshanski, G.I. Representations of infinite-dimensional classical groups, limits of envelopingalgebras, and Yangians. In Topics in Representation Theory; Kirillov, A.A., Ed.; Advances in Soviet Math; American Mathematical Society: Providence, RI, USA, 1991; pp. 1-66.

43. Okounkov, A.; Olshanski, G. Shifted Schur functions. Algebra i Analiz 1998, 9, 73-146.

44. Okounkov, A. Shifted Schur functions II. The binomial formulafor characters of classical groups and its applications. In American Mathematical Society Translations; v. 185, Kirillov's Seminar on Representation Theory; American Mathematical Society: Providence, RI, USA, 1998; pp. 245-271.

45. Okounkov, A. Quantum Immanants and Higher Capelli Identities; Transformation Groups, v. 1; Springer: Boston, MA, USA, 1996; pp. 99-126.

46. Okounkov, A. Young Basis, Wick Formula, and Higher Capelli Identities. Internat. Math. Res. Not. 1996, 17, 817-839. [CrossRef]

47. Vershik, A.M.; Okounkov, A.Y. A new approach to the representation theory of symmetric groups. II. J. Math. Sci. 2005, 131, 5471-5494. [CrossRef]

48. Gerasimov, A.A.; Shatashvili, S.L. Two-dimensional Gauge Theory and Quantum Integrable Systems. arXiv 2007, arXiv:0711.1472.

49. Rumanov, I. Classical integrability for beta ensembles and general Fokker-Plank equation. J. Math. Phys. 2015, 56, 013508. [CrossRef]

50. Mironov, A.; Morozov, A.; Semenoff, G. Unitary Matrix Integrals in the Framework of he Generalized Kontsevich Model. Intern J. Mod. Phys. A 1996, 11, 5031-5080. [CrossRef]

51. Jimbo, M.; Miwa, T. Solitons and Infinite Dimensional Lie Algebras. Publ. RIMS Kyoto Univ. 1983, 19, 943-1001. [CrossRef]

52. Ueno, K.; Takasaki, K. Toda lattice hierarchy. Adv. Stud. Pure Math. 1984, 4, 1-95.

53. Takasaki, K. Initial value problem for the Toda lattice hierarchy. Adv. Stud. Pure Math. 1984, 4, 139-163.

54. Takebe, T. Representation Theoretical Meaning of Initial Value Problem for the Toda Lattice Hierarchy I. Lett. Math. Phys. 1991, 21, 77-84. [CrossRef]

55. Takebe, T. Representation Theoretical Meaning of Initial Value Problem for the Toda Lattice Hierarchy II. Publ. RIMS Kyoto Univ. 1991, 27, 491-503.

56. Ekedahl, T.; Lando, S.; Shapiro, M.; Vainshtein, A. On Hurwitz numbers and Hodge integrals. Comptes Rendus de l'Académie des Sciences-Series I-Math. 1999, 146, 175-1180. [CrossRef] 
57. Okounkov, A. Toda equations for Hurwitz numbers. Math. Res. Lett. 2000, 7, 447-453. [CrossRef]

58. Mednykh, A.D. Determination of the number of nonequivalent covering over a compact Riemann surface. Soviet Math. Dokl. 1978, 19, 318-320.

59. Mednykh, A.D.; Pozdnyakova, G.G. The number of nonequivalent covering over a compact nonorientable surface. Sibirs. Mat. 1986, 27, 123-131. [CrossRef]

60. Jones, G.A. Enumeration of Homomorphisms and Surface-Coverings. Q. J. Math. Oxford 1995, 46, $485-507$. [CrossRef]

61. Mironov, A.D.; Morozov, A.Y.; Natanzon, S.M. Complect set of cut-and-join operators in the Hurwitz-Kontsevich theory. Theor. Math. Phys. 2011, 166, 1-22. [CrossRef]

62. Orlov, A.Y. Vertex Operator, $\bar{\partial}$-Problem, Symmetries, Variational Identities and Hamiltonian Formalism for $2+1$ Integrable Systems; Nonlinear and Turbulent Processes in Physics; World Scientific: Singapore, 1987.

63. Mironov, A.; Morozov, A.; Natanzon, S. Algebra of differential operators associated with Young diagramms. J. Geom. Phys. 2012, 62, 148-155. [CrossRef]

64. Perelomov, A.M.; Popov, V.S. Casimir operators for groups $U(N)$ SU(N). Yadernaya Fizika 1966, 3, $924-931$.

65. Perelomov, A.M.; Popov, V.S. Casimir operators for classical groups. Doklady AN SSSR 1967, 174, $287-290$. (In Russian)

66. Perelomov, A.M.; Popov, V.S. Casimir operators for semisimple Lie groups. Izavestia AN SSSR 1968, 32, 1368-1390. [CrossRef]

67. Zhelobenko, D.P. Compact Lie Groups and Their Representations; American Mathematical Soc.: Providence, RI, USA, 1973.

68. Dubrovin, B.A. Symplectic field theory of a disk, quantum integrable systems, and Schur polynomials. arXiv 2016, arXiv:1407.5824.

(C) 2020 by the authors. Licensee MDPI, Basel, Switzerland. This article is an open access article distributed under the terms and conditions of the Creative Commons Attribution (CC BY) license (http://creativecommons.org/licenses/by/4.0/). 